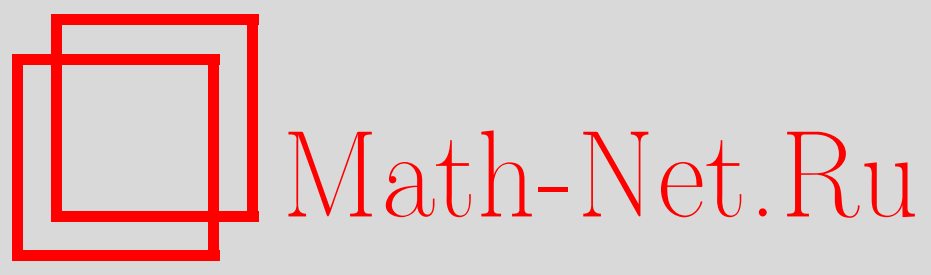

Н. А. Славнов, Алгебраический анзац Бете и квантовые интегрируемые системы, УМН, 2007, том 62, выпуск 4, 91-132

DOI: https://doi.org/10.4213/rm6847

Использование Общероссийского математического портала Math-Net.Ru подразумевает, что вы прочитали и согласны с пользовательским соглашением http://www . mathnet.ru/rus/agreement

Параметры загрузки:

IP : 54.198 .64 .247

26 апреля 2023 г., 14:49:54

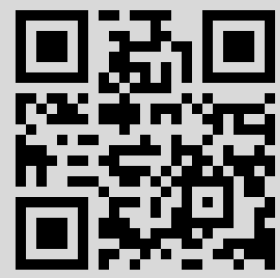




\section{Алгебраический анзац Бете и квантовые интегрируемые системы}

\section{Н. А. Славнов}

Рассматриваются методы применения алгебры с билинейными перестановочными соотношениями к теории квантовых интегрируемых систем. В обзоре собрано большинство результатов, полученных в этой области за последние 20 лет, применяющиеся в основном к вычислению корреляционных функций квантовых интегрируемых систем. Подробно излагаются методы построения собственных функций квантовой трансферматрицы, вычисления скалярных произведений и корреляционных функций. Рассмотрен пример применения общей схемы к модели $X X Z$-цепочки Гейзенберга.

Библиография: 51 название.

\section{СОДЕРЖАНИЕ}

1. Введение. . ......................................... 92

2. Квантовый метод обратной задачи ......................... 95

3. Спектр квантовомеханических гамильтонианов ................ 98

4. Собственные состояния трансферматрицы ................... 101

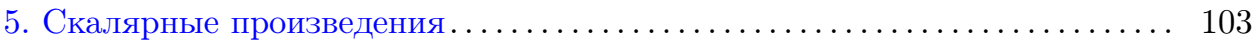

5.1. Выражение для общего коэффициента через старшие.......... 104

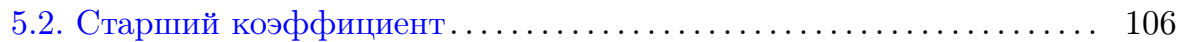

6. Частные случаи скалярного произведения ..................... 109

6.1. Вспомогательное тождество . . . . . . . . . . . . . . . . . . 110

6.2. Скалярное произведение собственного состояния на произвольное . . . . . . . . . . . . . . . . . . . . . . . . . . . . . . . 111

6.3. Ортогональность собственных функций ................. 112

6.4. Тождество для детерминантов ......................... 114

7. Двухкомпонентная модель . . . . . . . . . . . . . . . . . . . . 115

7.1. Определение двухкомпонентной модели . . . . . . . . . . . . 115

7.2. Матричный элемент оператора числа частиц на отрезке $[0, x] \ldots 117$

7.3. Частный случай матричного элемента оператора $Q_{1} \ldots \ldots \ldots \ldots$

Работа выполнена при поддержке научной программы Президиума РАН "Математические методы в нелинейной динамике", РФФИ (грант № 05-01-00498) и программы "Ведущие научные школы" (грант НШ-672.2006.1).

(C) Н. А. Славнов, 2007 
8. $X X Z$-цепочка Гейзенберга спина $1 / 2 \ldots \ldots \ldots \ldots \ldots \ldots \ldots \ldots \ldots \ldots \ldots \ldots \ldots \ldots \ldots \ldots$

9. Вероятность образования пустоты в пределе $M \rightarrow \infty \ldots \ldots \ldots \ldots \ldots \ldots . \ldots \ldots$

10. Вероятность образования пустоты при $\Delta=1 / 2 \ldots \ldots \ldots \ldots \ldots \ldots \ldots \ldots . \ldots \ldots$

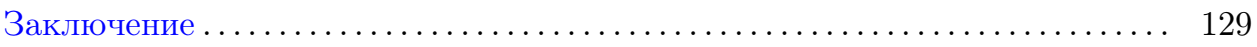

Список литературы ................................... 130

\section{1. Введение}

Настоящая работа представляет собой обзор некоторых результатов, полученных в области исследования квантовых интегрируемых систем. При решении всякой квантовомеханической задачи требуется ответить по крайней мере на два вопроса. Во-первых, необходимо найти спектр и систему собственных функций гамильтониана квантовой модели

$$
H|\psi\rangle=E|\psi\rangle .
$$

Во-вторых, требуется вычислить корреляционные функции операторов $\mathscr{O}$, соответствующих физическим наблюдаемым модели

$$
\langle O\rangle_{T}=\frac{\operatorname{tr}\left(\mathscr{O} e^{-H / T}\right)}{\operatorname{tr} e^{-H / T}},
$$

где $T$ - температура. В случае корреляционных функций при нулевой температуре последняя задача сводится к вычислению матричных элементов вида

$$
\langle O\rangle_{0}=\frac{\left\langle\psi_{g}|\mathscr{O}| \psi_{g}\right\rangle}{\left\langle\psi_{g} \mid \psi_{g}\right\rangle},
$$

где $\left|\psi_{g}\right\rangle$ - основное состояние гамильтониана.

Интегрируемые системы характеризуются тем свойством, что для них указанные задачи могут быть решены точно. Именно возможностью получения точных, непертурбативных результатов в таких моделях и объясняется интерес к ним.

В 1931 г. в работе [1] при решении весьма конкретной физической задачи был сформулирован метод (получивший название анзаца Бете), давший начало новому подходу к изучению целого класса квантовых интегрируемых систем. Этот метод позволил эффективно найти спектр квантовомеханических гамильтонианов (1.1) в таких моделях, как, например, спиновые цепочки Гейзенберга, одномерный Бозе-газ с точечным взаимодействием, массивная модель Тирринга и др. В последующие годы анзац Бете успешно применялся в целом ряде работ [2]-[12]. Несмотря на то, что модели, решаемые анзацем Бете, являются $(1+1)$-мерными, они находят достаточно широкое применение в различных областях квантовой физики, например, в физике твердого тела, моделях сверхпроводимости и нелинейной оптики.

К сожалению, метод, предложенный Бете, оказался малопригодным для вычисления корреляционных функций в этих моделях. До недавнего времени 
было известно лишь несколько результатов в этой области, касающихся в основном систем, эквивалентных свободным фермионам [13]-[21]. Основная проблема при вычислении корреляционных функций заключается в достаточно сложной структуре собственных функций гамильтониана $|\psi\rangle$, не позволяющей эффективно работать с этими выражениями.

В 1979 г. Л. Д. Фаддеевым, Е. К. Скляниным и Л. А. Тахтаджяном [22], [23] был сформулирован квантовый метод обратной задачи, одной из составных частей которого является алгебраическая версия анзаца Бете. По аналогии с классическим методом обратной задачи в квантовом случае совершается переход от исходных локальных операторов к данным рассеяния - матрице монодромии. Достоинством алгебраического анзаца Бете по сравнению с исходной версией этого метода является его универсальность. А именно, широкий класс моделей, имеющих совершенно различное физическое содержание, удается описать в рамках единой алгебры операторов, являющихся матричными элементами матрицы монодромии. При этом различные квантовые системы соответствуют различным представлениям этой алгебры. Как следствие, алгебраический анзац Бете позволяет единым образом строить систему собственных функций гамильтонианов сразу нескольких физических моделей.

Создание квантового метода обратной задачи послужило не только эффективным методом построения спектров квантовомеханических гамильтонианов. В ходе своего дальнейшего развития, осуществленного в основном представителями Ленинградской школы, этот метод послужил толчком для совершенно нового понимания взаимосвязи между квантовыми и классическими интегрируемыми системами. Можно без преувеличения сказать, что квантовый метод обратной задачи привел к созданию новой области современной математики теории квантовых групп (см. в том числе [24] и ссылки в этой работе). Мы однако не преследуем здесь целей дать наиболее полный исторический обзор результатов, связанных с этим методом, а напротив, стремимся ограничиться его применимостью к одной конкретной задаче - вычислению корреляционных функций.

K другим достоинствам алгебраического анзаца Бете следует отнести то, что этот метод позволяет наиболее строгим образом решить проблему расходимостей, которые, как правило, возникают в квантовой теории. Существующие альтернативные методы в основном имеют дело с квантовыми системами, изначально находящимися в бесконечном объеме, что приводит к ненормируемости собственных функций гамильтониана. Напротив, алгебраический анзац Бете имеет дело с системами в конечном объеме, что позволяет нормировать собственные функции, а переход к бесконечный объему (термодинамическому пределу) выполнить уже на стадии вычисления матричных элементов операторов.

Следует заметить, что если изначально алгебраическим анзацем Бете назывался лишь метод построения собственных состояний гамильтонианов, то впоследствии это понятие расширилось и стало применяться к любым вычислениям в алгебре матричных элементов матрицы монодромии. Естественно, 
универсальность этого метода в задаче о собственных функциях породила желание воспользоваться такой универсальностью для вычисления корреляционных функций. В этом вопросе, однако, как и в первоначальной версии анзаца Бете, возникают серьезные сложности, но уже по иным причинам. Первая и главная проблема состоит в том, как включить локальные операторы исходной модели, зависящие от конкретной точки пространства-времени, в алгебру операторов матрицы монодромии, которая не является локальным объектом, поскольку относится ко всей системе в целом. Ответом на этот вопрос явилось бы явное решение квантовой обратной задачи, однако на сегодняшний день такое решение известно лишь для моделей спиновых цепочек Гейзенберга [25]. Есть серьезные основания считать, что если в общем случае подобное решение и существует, то вряд ли оно может быть использовано для практических вычислений.

Указанная проблема не исчерпывает список трудностей, возникающих при применении алгебраического анзаца Бете к вычислению корреляционных функций. В ряде случаев удается вычислить действие локальных операторов на собственные состояния гамильтониана, не прибегая к решению квантовой обратной задачи. С формальной точки зрения после этого для решения задачи остается лишь использовать коммутационные соотношения в алгебре матричных элементов матрицы монодромии. Однако эти перестановочные соотношения достаточно сложны, и в результате получающиеся ответы оказываются настолько громоздкими, что никакой физически интересной информации получить из них невозможно.

Если сформулировать цель настоящей статьи более конкретно, то она состоит как раз в том, чтобы продемонстрировать, как следует работать с упомянутыми перестановочными соотношениями, чтобы в конечном итоге получить такие представления для корреляционных функций, из которых уже можно получить физические характеристики исследуемых квантовых систем. Основные правила, которых следует придерживаться при вычислениях в алгебре матричных элементов матрицы монодромии, были сформулированы еще в первых работах по квантовому методу обратной задачи [22], [23], [26]-[30]. История квантового метода обратной задачи и соответствующая литература представлены в работе Л.Д. Фаддеева [31]. В 1993 г. многие из результатов, полученных на тот момент в области применения алгебраического анзаца Бете, были подробно отражены в книге [32]. C тех пор, однако, произошло весьма существенное развитие. В частности, был установлен ряд нетривиальных алгебраических тождеств, позволяющих получить новые результаты, а также существенно упростить старые. Это, в свою очередь, позволило построить интегральные представления для корреляционных функций в ряде физических моделей. В настоящей работе приводится подробный обзор этих результатов.

План работы следующий. Во втором разделе мы вводим основные понятия квантового метода обратной задачи. В третьем разделе вычисляется действие матричных элементов матрицы монодромии на векторы пространства состояний. В разделе 4 описана схема построения собственных состояний квантовой 
трансферматрицы. Пятый раздел посвящен вычислению скалярных произведений, частные случаи которых рассмотрены в шестом разделе. В седьмом разделе вводится двухкомпонентная обобщенная модель, необходимая для вычисления ряда корреляционных функций. Последние три раздела посвящены применению общей схемы к конкретной модели $X X Z$-цепочки Гейзенберга. В восьмом разделе дано конкретное представление алгебры, описывающее $X X Z$-цепочки. $\mathrm{B}$ девятом разделе получено интегральное представление для специальной корреляционной функции, называющейся вероятностью образования пустоты. В десятом разделе вероятность образования пустоты вычислена при значении параметра анизотропии, равного $1 / 2$.

\section{2. Квантовый метод обратной задачи}

В этом разделе мы вводим основные понятия и обозначения квантового метода обратной задачи. При этом исходными для нас являются данные обратной задачи и соответствующая алгебра, а конкретные физические интегрируемые системы возникают как следствие этой алгебры. Мы не преследуем здесь цели дать максимально общее описание метода, а сознательно ограничиваемся лишь моделями, имеющими $R$-матрицу специального вида (см. ниже). Более подробное описание квантового метода обратной задачи можно найти в [24], [32].

Рассмотрим $(2 \times 2)$-матрицу $T(\lambda)$, которую мы будем называть матрицей монодромии [22], [23]:

$$
T(\lambda)=\left(\begin{array}{ll}
A(\lambda) & B(\lambda) \\
C(\lambda) & D(\lambda)
\end{array}\right) .
$$

Матричные элементы $A, B, C$ и $D$ зависят от комплексного параметра $\lambda$ и являются операторами, действующими в некотором гильбертовом пространстве $\mathscr{H}$. Допустим, что коммутационные соотношения между $T_{j k}(\lambda)$ заданы в виде

$$
R_{12}(\lambda-\mu) T_{1}(\lambda) T_{2}(\mu)=T_{2}(\mu) T_{1}(\lambda) R_{12}(\lambda-\mu) .
$$

Объясним обозначения, использованные в (2.2). Равенство (2.2) имеет место в прямом произведении трех пространств $V_{1} \otimes V_{2} \otimes \mathscr{H}$, где $V_{j} \sim \mathbb{C}^{2}$. При этом $T_{1}(\lambda)=T(\lambda) \otimes I$ нетривиально действует в пространстве $V_{1} \otimes \mathscr{H}$, a $T_{2}(\lambda)=I \otimes T(\lambda)$ нетривиально действует в пространстве $V_{2} \otimes \mathscr{H}$. Матрица $R_{12}(\lambda)$ является числовой матрицей размера $4 \times 4$ и действует нетривиально в пространстве $V_{1} \otimes V_{2}$. Соотношение (2.2) задает алгебру с билинейными перестановочными соотношениями, в которой $R$-матрица является аналогом структурных констант.

Будем считать, что $R$-матрица почти всюду обратима. Тогда из соотношения (2.2) немедленно следует, что

$$
[\mathscr{T}(\lambda), \mathscr{T}(\mu)]=0,
$$

где

$$
\mathscr{T}(\lambda)=\operatorname{tr} T(\lambda)=A(\lambda)+D(\lambda) .
$$


Раскладывая теперь $\mathscr{T}(\lambda)$ в степенной ряд с центром в некоторой точке $\lambda_{0}$ :

$$
\mathscr{T}(\lambda)=\sum_{n}\left(\lambda-\lambda_{0}\right)^{n} I_{n},
$$

мы получаем набор коммутирующих операторов $I_{n}:\left[I_{n}, I_{m}\right]=0$. Если теперь мы примем один из этих операторов в качестве гамильтониана квантовомеханической системы, мы тем самым построим модель, обладающую, вообще говоря, бесконечным набором законов сохранения, т. е. интегрируемую систему.

Описанная выше схема построения квантовой интегрируемой системы является, разумеется, всего лишь общим рецептом, применимость которого следует отдельно обосновывать в каждом конкретном случае. Так, например, построенные законы сохранения $I_{n}$ могут оказаться тривиальными или функционально зависимыми. Кроме того, в моделях, имеющих физическое содержание, операторы $I_{n}$ должны обладать свойством локальности, которое никак не следует из приведенного выше построения. Тем не менее, большое количество физически интересных квантовых интегрируемых систем, таких, например, как квантовый одномерный Бозе-газ, спиновые цепочки Гейзенберга, модель синус-Гордон и др., могут быть построены именно с помощью описанной процедуры [22], [23], [33], [34]. В этом случае изучение спектра и корреляционных функций таких моделей сводится к различного рода вычислениям в алгебре (2.2).

Соотношение (2.2) накладывает некоторые ограничения на $R$-матрицу. Рассмотрим произведение трех матриц монодромии $T_{1}\left(\lambda_{1}\right) T_{2}\left(\lambda_{2}\right) T_{3}\left(\lambda_{3}\right)$ и изменим их порядок на обратный $T_{3}\left(\lambda_{3}\right) T_{2}\left(\lambda_{2}\right) T_{1}\left(\lambda_{1}\right)$, пользуясь соотношением $(2.2)$. Очевидно, что существуют два способа сделать это:

$$
\begin{aligned}
& T_{1} T_{2} T_{3} \rightarrow T_{2} T_{1} T_{3} \rightarrow T_{2} T_{3} T_{1} \rightarrow T_{3} T_{2} T_{1}, \\
& T_{1} T_{2} T_{3} \rightarrow T_{1} T_{3} T_{2} \rightarrow T_{3} T_{1} T_{2} \rightarrow T_{3} T_{2} T_{1} .
\end{aligned}
$$

Достаточным условием совместности этих двух способов перестановки является следующее соотношение на $R$-матрицу [22], [23], называемое уравнением Янга-Бакстера:

$$
R_{12}\left(\lambda_{1}-\lambda_{2}\right) R_{13}\left(\lambda_{1}-\lambda_{3}\right) R_{23}\left(\lambda_{2}-\lambda_{3}\right)=R_{23}\left(\lambda_{2}-\lambda_{3}\right) R_{13}\left(\lambda_{1}-\lambda_{3}\right) R_{12}\left(\lambda_{1}-\lambda_{2}\right) .
$$

Данное уравнение выполняется в прямом произведении трех пространств $V_{1} \otimes$ $V_{2} \otimes V_{3}$, при этом индексы у $R$-матриц указывают на ту пару пространств, в которой данная $R$-матрица действует нетривиально.

Уравнение Янга-Бакстера является матричным функциональным уравнением. Одним из его простейших решений является $R$-матрица шестивершинной модели

$$
R(\lambda-\mu)=\left(\begin{array}{cccc}
f(\lambda, \mu) & 0 & 0 & 0 \\
0 & 1 & g(\lambda, \mu) & 0 \\
0 & g(\lambda, \mu) & 1 & 0 \\
0 & 0 & 0 & f(\lambda, \mu)
\end{array}\right)
$$



где

$$
f(\lambda, \mu)=\frac{\operatorname{sh}(\lambda-\mu+\eta)}{\operatorname{sh}(\lambda-\mu)}, \quad g(\lambda, \mu)=\frac{\operatorname{sh} \eta}{\operatorname{sh}(\lambda-\mu)} .
$$

Величина $\eta$ является произвольной комплексной константой, которая, как правило, связана с параметрами квантовомеханического гамильтониана (константа связи, параметр анизотропии и т. п.). Матрицу (2.8) с матричными элементами (2.9) часто называют тригонометрической $R$-матрицей. В вырожденном случае

$$
\lambda \rightarrow \epsilon \lambda, \quad \mu \rightarrow \epsilon \mu, \quad \eta \rightarrow \epsilon \eta, \quad \epsilon \rightarrow 0
$$

тригонометрическая $R$-матрица переходит в рациональную с элементами

$$
f(\lambda, \mu)=\frac{\lambda-\mu+\eta}{\lambda-\mu}, \quad g(\lambda, \mu)=\frac{\eta}{\lambda-\mu} .
$$

Все дальнейшее изложение будет вестись одновременно для обоих случаев. При этом, кроме случаев, оговоренных особо, мы будем использовать обозначения $f(\lambda, \mu)$ и $g(\lambda, \mu)$, не конкретизируя, к какой $R$-матрице, тригонометрической или рациональной, они относятся. В частности, в дальнейшем для сокращения записи мы часто будем использовать функции

$$
h(\lambda, \mu)=\frac{f(\lambda, \mu)}{g(\lambda, \mu)}, \quad t(\lambda, \mu)=\frac{g^{2}(\lambda, \mu)}{f(\lambda, \mu)},
$$

которые равны

$$
h(\lambda, \mu)=\frac{\operatorname{sh}(\lambda-\mu+\eta)}{\operatorname{sh} \eta}, \quad t(\lambda, \mu)=\frac{(\operatorname{sh} \eta)^{2}}{\operatorname{sh}(\lambda-\mu) \operatorname{sh}(\lambda-\mu+\eta)}
$$

для тригонометрической $R$-матрицы и

$$
h(\lambda, \mu)=\frac{\lambda-\mu+\eta}{\eta}, \quad t(\lambda, \mu)=\frac{\eta^{2}}{(\lambda-\mu)(\lambda-\mu+\eta)}
$$

для рациональной.

Для полного задания квантовомеханической системы необходимо описать пространство состояний ее гамильтониана. В рамках квантового метода обратной задачи это делается следующим образом. Будем считать, что гильбертово пространство $\mathscr{H}$, в котором действуют операторы $T_{j k}(\lambda)$, имеет структуру фоковского пространства. А именно, существует циклический вектор $|0\rangle$, называемый псевдовакуумом, который является собственным для операторов $A$ и $D$ и аннигилируется оператором $C$ :

$$
A(\lambda)|0\rangle=a(\lambda)|0\rangle, \quad D(\lambda)|0\rangle=d(\lambda)|0\rangle, \quad C(\lambda)|0\rangle=0 .
$$

Здесь $a(\lambda)$ и $d(\lambda)$ - некоторые комплекснозначные функции, явный вид которых зависит от конкретной модели. Все пространство состояний представ- 
ляет собой замыкание линейной оболочки векторов вида $\prod_{j=1}^{N} B\left(\lambda_{j}\right)|0\rangle, N=$ $0,1,2, \ldots$. Дуальные состояния строятся аналогичным образом с помощью оператора $C:\langle 0| \prod_{j=1}^{N} C\left(\lambda_{j}\right)$, где дуальный псевдовакуум обладает свойствами

$$
\langle 0| A(\lambda)=a(\lambda)\langle 0|, \quad\langle 0| D(\lambda)=d(\lambda)\langle 0|, \quad\langle 0| B(\lambda)=0 .
$$

Выбор конкретных вакуумных собственных значений $a(\lambda)$ и $d(\lambda)$ равносилен выбору конкретного представления алгебры (2.2). Замечательным, однако, является тот факт, что большое количество результатов может быть получено уже на чисто алгебраическом уровне, без учета конкретного представления. В настоящей работе мы в основном сосредоточимся именно на таких результатах. Будем называть модель, определенную соотношениями (2.1), (2.8), (2.2), (2.15) и (2.16), обобщенной моделью [28], в которой зафиксирована лишь $R$-матрица $(2.8)$, а функции $a(\lambda)$ и $d(\lambda)$ играют роль свободных функциональных параметров.

\section{3. Спектр квантовомеханических гамильтонианов}

В силу (2.5) собственные функции квантовомеханического гамильтониана и коммутирующих с ним интегралов движения совпадают с собственными функциями трансферматрицы $\mathscr{T}(\lambda)=A(\lambda)+D(\lambda)$. Для их построения необходимо вычислить действие операторов $A$ и $D$ на состояния вида $\prod_{j=1}^{N} B\left(\lambda_{j}\right)|0\rangle$. Для этого, в свою очередь, нам потребуется явный вид коммутационных соотношений (2.2). Приведем полный список перестановочных соотношений для матричных элементов $T_{j k}(\lambda)$ :

$$
\begin{aligned}
{\left[T_{j k}(\lambda), T_{j k}(\mu)\right] } & =0, \quad j, k=1,2, \\
A(\mu) B(\lambda) & =f(\lambda, \mu) B(\lambda) A(\mu)+g(\mu, \lambda) B(\mu) A(\lambda), \\
B(\mu) A(\lambda) & =f(\lambda, \mu) A(\lambda) B(\mu)+g(\mu, \lambda) A(\mu) B(\lambda), \\
D(\mu) B(\lambda) & =f(\mu, \lambda) B(\lambda) D(\mu)+g(\lambda, \mu) B(\mu) D(\lambda), \\
B(\mu) D(\lambda) & =f(\mu, \lambda) D(\lambda) B(\mu)+g(\lambda, \mu) D(\mu) B(\lambda), \\
A(\mu) C(\lambda) & =f(\mu, \lambda) C(\lambda) A(\mu)+g(\lambda, \mu) C(\mu) A(\lambda), \\
C(\mu) A(\lambda) & =f(\mu, \lambda) A(\lambda) C(\mu)+g(\lambda, \mu) A(\mu) C(\lambda), \\
D(\mu) C(\lambda) & =f(\lambda, \mu) C(\lambda) D(\mu)+g(\mu, \lambda) C(\mu) D(\lambda), \\
C(\mu) D(\lambda) & =f(\lambda, \mu) D(\lambda) C(\mu)+g(\mu, \lambda) D(\mu) C(\lambda), \\
{[C(\mu), B(\lambda)] } & =g(\lambda, \mu)(A(\mu) D(\lambda)-A(\lambda) D(\mu)) \\
& =g(\lambda, \mu)(D(\lambda) A(\mu)-D(\mu) A(\lambda)), \\
{[D(\mu), A(\lambda)] } & =g(\lambda, \mu)(B(\mu) C(\lambda)-B(\lambda) C(\mu)) \\
& =g(\lambda, \mu)(C(\lambda) B(\mu)-C(\mu) B(\lambda)) .
\end{aligned}
$$

Действие операторов на состояния. Вычислим действие оператора $A(\mu)$ на вектор $\prod_{j=1}^{N} B\left(\lambda_{j}\right)|0\rangle$, предполагая, что параметры $\mu, \lambda_{1}, \ldots, \lambda_{N}$ суть комплексные числа в общем положении [22], [23]. Формально результат можно 
получить, применяя $N$ раз формулу (3.2). При этом на каждом шаге мы получим два слагаемых, и в итоге результат будет содержать $2^{N}$ слагаемых. Легко видеть, однако, что на самом деле число линейно независимых слагаемых в конечном ответе во всяком случае не превосходит $N+1$,

$$
\begin{aligned}
A(\mu) \prod_{j=1}^{N} B\left(\lambda_{j}\right)|0\rangle=a(\mu) \Lambda(\mu \mid\{\lambda\}) \prod_{j=1}^{N} B\left(\lambda_{j}\right)|0\rangle & \\
& +\sum_{k=1}^{N} a\left(\lambda_{k}\right) \Lambda_{k}(\mu \mid\{\lambda\}) B(\mu) \prod_{\substack{j=1 \\
j \neq k}}^{N} B\left(\lambda_{j}\right)|0\rangle .
\end{aligned}
$$

Действительно, из формулы (3.2) следует, что при перестановке операторов $A$ и $B$ последние либо сохраняют свои аргументы, либо обмениваются ими, однако в любом случае действует “закон сохранения аргументов”. Поэтому если в левой части формулы (3.12) аргументами операторов $A$ и $B$ являются числа $\mu, \lambda_{1}, \ldots, \lambda_{N}$, то и в правой части мы должны иметь те же самые аргументы операторов. Формула (3.12) представляет собой наиболее общий вид такого равенства с учетом (2.15). Коэффициенты $\Lambda$ и $\Lambda_{k}$, возникающие при коммутации, зависят только от $R$-матрицы и подлежат определению.

Будем называть первое слагаемое в правой части соотношения (3.2) первой схемой коммутации (операторы сохраняют свои аргументы), а второе слагаемое второй схемой коммутации (операторы обмениваются аргументами). Вычислим коэффициент $\Lambda$. Очевидно, что соответствующий вклад в (3.12) может возникнуть только при условии, что, переставляя операторы $A(\mu)$ и $B\left(\lambda_{j}\right)$, мы каждый раз будем пользоваться только первой схемой коммутации. Тогда, действуя на псевдовакуум, оператор $A(\mu)$ даст функцию $a(\mu)$, а коэффициент, возникающий из перестановочных соотношений, оказывается равным

$$
\Lambda(\mu \mid\{\lambda\})=\prod_{j=1}^{N} f\left(\lambda_{j}, \mu\right) .
$$

Для того чтобы найти коэффициенты $\Lambda_{k}$, зафиксируем некоторое $k$ и, воспользовавшись соотношениями (3.1), перепишем левую часть формулы (3.12) в виде

$$
A(\mu) \prod_{j=1}^{N} B\left(\lambda_{j}\right)|0\rangle=A(\mu) B\left(\lambda_{k}\right) \prod_{\substack{j=1 \\ j \neq k}}^{N} B\left(\lambda_{j}\right)|0\rangle .
$$

Теперь для того, чтобы получить вклад, пропорциональный $a\left(\lambda_{k}\right)$, необходимо на первом шаге при перестановке операторов $A(\mu)$ и $B\left(\lambda_{k}\right)$ воспользоваться второй схемой. В противном случае при дальнейшем движении оператора $A$ направо мы уже не сможем получить $A\left(\lambda_{k}\right)$, а следовательно, и $a\left(\lambda_{k}\right)$. Таким образом,

$$
A(\mu) B\left(\lambda_{k}\right) \prod_{\substack{j=1 \\ j \neq k}}^{N} B\left(\lambda_{j}\right)|0\rangle=g\left(\mu, \lambda_{k}\right) B(\mu) A\left(\lambda_{k}\right) \prod_{\substack{j=1 \\ j \neq k}}^{N} B\left(\lambda_{j}\right)|0\rangle+\mathscr{Z},
$$


где символом $\mathscr{Z}$ обозначено слагаемое, заведомо не дающее вклад в искомый коэффициент. Легко видеть, что теперь при дальнейшем перемещении направо оператор $A\left(\lambda_{k}\right)$ должен всегда сохранять свой аргумент, т. е. мы снова должны использовать только первую схему коммутации. В результате находим

$$
\Lambda_{k}(\mu \mid\{\lambda\})=g\left(\mu, \lambda_{k}\right) \prod_{\substack{j=1 \\ j \neq k}}^{N} f\left(\lambda_{j}, \lambda_{k}\right) .
$$

Полностью аналогично вычисляется действие оператора $D(\mu)$ :

$$
\begin{aligned}
D(\mu) \prod_{j=1}^{N} B\left(\lambda_{j}\right)|0\rangle=d(\mu) & \bar{\Lambda}(\mu \mid\{\lambda\}) \prod_{j=1}^{N} B\left(\lambda_{j}\right)|0\rangle \\
& +\sum_{k=1}^{N} d\left(\lambda_{k}\right) \bar{\Lambda}_{k}(\mu \mid\{\lambda\}) B(\mu) \prod_{\substack{j=1 \\
j \neq k}}^{N} B\left(\lambda_{j}\right)|0\rangle,
\end{aligned}
$$

где

$$
\bar{\Lambda}(\mu \mid\{\lambda\})=\prod_{j=1}^{N} f\left(\mu, \lambda_{j}\right), \quad \bar{\Lambda}_{k}(\mu \mid\{\lambda\})=g\left(\lambda_{k}, \mu\right) \prod_{\substack{j=1 \\ j \neq k}}^{N} f\left(\lambda_{k}, \lambda_{j}\right) .
$$

На первый взгляд может показаться странным, что функция $g$ входит в конечные ответы (3.12), (3.17) только линейно. Исходя из коммутационных соотношений (3.2), (3.4), мы могли бы ожидать, что зависимость конечных результатов от $g$ будет полиномиальной, вплоть до членов, пропорциональных $g^{N}$. Причина этой странности кроется в том, что благодаря уравнению Янга-Бакстера функции $f$ и $g$ оказываются связанными между собой рядом функциональных тождеств. Легко проверить, что если мы не наложим никаких ограничений на эти функции, то коммутационные соотношения (3.2), (3.4) и $[B(\lambda), B(\mu)]=0$ окажутся несовместными. В случае конкретных функций $(2.9)$ (или (2.11)) совместность гарантируется уравнением (2.7).

В разделе 5 нам также потребуется явная формула для действия оператора $C(\mu)$ на вектор $\prod_{j=1}^{N} B\left(\lambda_{j}\right)|0\rangle[28],[29]$ :

$$
C(\mu) \prod_{j=1}^{N} B\left(\lambda_{j}\right)|0\rangle=\sum_{\ell=1}^{N} M_{\ell} \prod_{\substack{j=1 \\ j \neq \ell}}^{N} B\left(\lambda_{j}\right)|0\rangle+\sum_{\ell>m}^{N} M_{\ell m} B(\mu) \prod_{\substack{j=1 \\ j \neq \ell, m}}^{N} B\left(\lambda_{j}\right)|0\rangle,
$$

где

$$
\begin{aligned}
M_{\ell}=g\left(\lambda_{\ell}, \mu\right) d\left(\lambda_{\ell}\right) a(\mu) & \prod_{\substack{j=1 \\
j \neq \ell}}^{N} f\left(\lambda_{\ell}, \lambda_{j}\right) f\left(\lambda_{j}, \mu\right) \\
& +g\left(\mu, \lambda_{\ell}\right) d(\mu) a\left(\lambda_{\ell}\right) \prod_{\substack{j=1 \\
j \neq \ell}}^{N} f\left(\lambda_{j}, \lambda_{\ell}\right) f\left(\mu, \lambda_{j}\right),
\end{aligned}
$$




$$
\begin{aligned}
M_{\ell m}=d\left(\lambda_{\ell}\right) a\left(\lambda_{m}\right) g\left(\lambda_{\ell}, \mu\right) g\left(\mu, \lambda_{m}\right) f\left(\lambda_{\ell}, \lambda_{m}\right) & \prod_{\substack{j=1 \\
j \neq \ell, m}}^{N} f\left(\lambda_{\ell}, \lambda_{j}\right) f\left(\lambda_{j}, \lambda_{m}\right) \\
& +d\left(\lambda_{m}\right) a\left(\lambda_{\ell}\right) g\left(\lambda_{m}, \mu\right) g\left(\mu, \lambda_{\ell}\right) f\left(\lambda_{m}, \lambda_{\ell}\right) \prod_{\substack{j=1 \\
j \neq \ell, m}}^{N} f\left(\lambda_{m}, \lambda_{j}\right) f\left(\lambda_{j}, \lambda_{\ell}\right) .
\end{aligned}
$$

Кратко поясним вывод формул (3.20), (3.21), который, впрочем, вполне аналогичен выводу формул для коэффициентов в равенствах (3.12), (3.17). Из коммутационных соотношений (3.10) следует, что при действии оператора $C$ возникают слагаемые трех типов: $d\left(\lambda_{\ell}\right) a(\mu), a\left(\lambda_{\ell}\right) d(\mu)$ и $d\left(\lambda_{\ell}\right) a\left(\lambda_{m}\right)$. Для того чтобы получить, например, коэффициент при $d\left(\lambda_{\ell}\right) a(\mu)$, следует переупорядочить операторы $B$ так, чтобы оператор $B\left(\lambda_{\ell}\right)$ находился в крайнем левом положении. После этого становится очевидным, что искомый коэффициент возникает при единственном способе коммутации операторов. Аналогично находятся все остальные коэффициенты.

Точно так же можно вычислить действие операторов $A, D$ и $B$ на дуальные состояния $\langle 0| \prod_{j=1}^{N} C\left(\lambda_{j}\right)$. Получающиеся при этом формулы полностью аналогичны приведенным выше, причем все коэффициенты по-прежнему задаются формулами (3.13), (3.16), (3.18), (3.20), (3.21).

\section{4. Собственные состояния трансферматрицы}

Метод алгебраического анзаца Бете состоит в том, что собственные состояния трансферматрицы ищутся в виде

$$
|\psi(\{\lambda\})\rangle=\prod_{j=1}^{N} B\left(\lambda_{j}\right)|0\rangle .
$$

Допустим, что все параметры $\lambda_{k}$ попарно различны: $\lambda_{j} \neq \lambda_{k}, j, k=1, \ldots, N$. Тогда из соотношений (3.12), (3.17) следует, что если

$$
a\left(\lambda_{k}\right) \Lambda_{k}(\mu \mid\{\lambda\})+d\left(\lambda_{k}\right) \bar{\Lambda}_{k}(\mu \mid\{\lambda\})=0 \quad \forall \mu, \quad k=1, \ldots, N,
$$

то вектор $|\psi(\{\lambda\})\rangle$ является собственным вектором оператора $\mathscr{T}(\mu)$,

$$
\mathscr{T}(\mu)|\psi(\{\lambda\})\rangle=\tau(\mu \mid\{\lambda\}) \cdot|\psi(\{\lambda\})\rangle,
$$

с собственным значением

$$
\tau(\mu \mid\{\lambda\})=a(\mu) \prod_{a=1}^{N} f\left(\lambda_{a}, \mu\right)+d(\mu) \prod_{a=1}^{N} f\left(\mu, \lambda_{a}\right) .
$$

Введем функцию

$$
\mathscr{Y}(\mu \mid\{\lambda\})=\tau(\mu \mid\{\lambda\}) \prod_{a=1}^{N} g^{-1}\left(\lambda_{a}, \mu\right)=a(\mu) \prod_{a=1}^{N} h\left(\lambda_{a}, \mu\right)+(-1)^{N} d(\mu) \prod_{a=1}^{N} h\left(\mu, \lambda_{a}\right) .
$$


Тогда условие (4.2) эквивалентно системе уравнений

$$
\mathscr{Y}\left(\lambda_{k} \mid\{\lambda\}\right)=0, \quad k=1, \ldots, N,
$$

называемой уравнениями Бете.

Отметим, что вывод системы уравнений (4.6) в рамках алгебраического анзаца Бете носит универсальный характер. Для получения этой системы используются только перестановочные соотношения в алгебре (2.2). Такая универсальность, безусловно, является достоинством данного метода, но вместе с тем она же является и его недостатком. Во-первых, не зная конкретного представления алгебры (2.2), мы не можем утверждать, что состояния, отвечающие различным наборам параметров $\{\lambda\}$, являются линейно независимыми. Следовательно, равенство (4.2), а значит, и система (4.6) не являются необходимым условием. Во-вторых, без знания конкретного представления алгебры (2.2) мы ничего не можем сказать о полноте построенной системы состояний. Таким образом, мы можем утверждать лишь то, что алгебраический анзац Бете позволяет построить систему состояний, претендующую на право быть полной системой собственных функций трансферматрицы. Вопрос о том, насколько эти претензии обоснованы, выходит за рамки данного метода и должен решаться отдельно в каждом конкретном случае.

Формально, уравнения Бете могут иметь решения, в которых несколько параметров $\lambda_{j}$ совпадают. Несложно проверить, однако, что в этом случае система (4.6) и равенство (4.2) не эквивалентны. Строго говоря, формулы (3.16), (3.18) были получены в предположении, что все параметры $\lambda_{k}$ попарно различны. Если некоторые из этих параметров совпадают, то коэффициенты $\Lambda_{k}(\mu \mid\{\lambda\})$ и $\bar{\Lambda}_{k}(\mu \mid\{\lambda\})$ становятся сингулярными, хотя формулы для действия операторов $A$ и $D$ (3.12), (3.17) по-прежнему сохраняют смысл. В этом случае в ответ для действия трансферматрицы на вектор состояния входят производные от коэффициентов $\Lambda_{k}(\mu \mid\{\lambda\})$ и $\bar{\Lambda}_{k}(\mu \mid\{\lambda\})$, а также производные от операторов $B$. Если мы потребуем, чтобы такой вектор был собственным вектором трансферматрицы, то получим новую систему, которая будет по-прежнему содержать $N$ уравнений, но число неизвестных будет уже меньше $N$ [35]. В моделях, описывающих реальные физические системы, такая переопределенная система не имеет решений, и в дальнейшем мы будем предполагать, что в собственных состояниях $|\psi(\{\lambda\})\rangle$ все параметры $\lambda_{j}$ различны.

При вычислении некоторых корреляционных функций часто бывает полезно ввести в систему уравнений Бете дополнительный комплексный параметр $\varkappa[36],[37]:$

$$
\mathscr{Y}_{\varkappa}\left(\lambda_{k} \mid\{\lambda\}\right)=0, \quad k=1, \ldots, N
$$

где

$$
\mathscr{Y}_{\varkappa}(\mu \mid\{\lambda\})=a(\mu) \prod_{a=1}^{N} h\left(\lambda_{a}, \mu\right)+(-1)^{N} \varkappa d(\mu) \prod_{a=1}^{N} h\left(\mu, \lambda_{a}\right) .
$$

Возможность и естественность такого обобщения вытекает из следующего простого наблюдения. 
Пусть $\omega$ является $(2 \times 2)$-матрицей такой, что ее тензорный квадрат коммутирует с $R$-матрицей: $\left[\omega_{1} \omega_{2}, R_{12}\right]=0$. Пусть далее $T(\lambda)$ удовлетворяет соотношению (2.2). Тогда можно легко убедиться в том, что матрица $\widetilde{T}(\lambda)=\omega T(\lambda)$ также удовлетворяет соотношению (2.2).

В случае $R$-матрицы (2.8) матрицу $\omega$ можно выбрать в виде

$$
\omega=\left(\begin{array}{ll}
1 & 0 \\
0 & \varkappa
\end{array}\right)
$$

Тогда все приведенные выше построения остаются справедливыми и для матрицы монодромии

$$
\widetilde{T}(\lambda)=\omega T(\lambda)=\left(\begin{array}{cc}
A(\lambda) & B(\lambda) \\
\varkappa C(\lambda) & \varkappa D(\lambda)
\end{array}\right)
$$

В частности, собственные функции оператора $\mathscr{T}_{\varkappa}(\mu)=A(\mu)+\varkappa D(\mu)$, который мы будем называть твистованной трансферматрицей, имеют вид $\prod_{j=1}^{N} B\left(\lambda_{j}\right)|0\rangle$ при условии, что параметры $\{\lambda\}$ являются решениями твистованной системы уравнений Бете (4.7). При этом соответствующие собственные значения равны

$$
\tau_{\varkappa}(\mu \mid\{\lambda\})=a(\mu) \prod_{a=1}^{N} f\left(\lambda_{a}, \mu\right)+\varkappa d(\mu) \prod_{a=1}^{N} f\left(\mu, \lambda_{a}\right) .
$$

Собственные функции твистованной трансферматрицы будут обозначаться

$$
\left|\psi_{\varkappa}(\{\lambda\})\right\rangle=\prod_{j=1}^{N} B\left(\lambda_{j}\right)|0\rangle .
$$

Полностью аналогично рассматривается задача о собственных функциях трансферматрицы в пространстве дуальных состояний. Так, дуальные собственные функции оператора $\mathscr{T}_{\varkappa}$ (в том числе и при $\left.\varkappa=1\right)$ имеют вид

$$
\left\langle\psi_{\varkappa}(\{\lambda\})\right|=\langle 0| \prod_{j=1}^{N} C\left(\lambda_{j}\right)
$$

при условии, что параметры $\{\lambda\}$ удовлетворяют системе (4.7). При этом собственные значения по-прежнему даются формулой (4.11).

\section{5. Скалярные произведения}

В этом разделе мы рассмотрим скалярные произведения

$$
S_{N}(\{\mu\} \mid\{\lambda\})=\left\langle 0\left|\prod_{j=1}^{N} C\left(\mu_{j}\right) \prod_{j=1}^{N} B\left(\lambda_{j}\right)\right| 0\right\rangle,
$$

где параметры $\mu_{1}, \ldots, \mu_{N}$ и $\lambda_{1}, \ldots, \lambda_{N}$ являются произвольными комплексными числами и, вообще говоря, не удовлетворяют никаким дополнительным ограничениям. С формальной точки зрения у нас есть все необходимое для вы- 
числения этого объекта, а именно коммутационные соотношения (3.1)-(3.10) и формулы (2.15), (2.16). Несложно, например, вычислить

$$
S_{1}(\mu \mid \lambda)=g(\lambda, \mu)(a(\mu) d(\lambda)-a(\lambda) d(\mu))
$$

Легко видеть, однако, что число слагаемых в конечном результате экспоненциально растет с ростом $N$, поэтому вычисление скалярных произведений (5.1) для произвольного $N$ далеко от тривиального.

Ясно, что после применения коммутационных соотношений (3.10) параметры $\{\mu\}$ и $\{\lambda\}$ сначала становятся аргументами операторов $A$ и $D$, а затем, в силу $(3.2),(3.4)$ и (2.15), они становятся аргументами вакуумных собственных значений $a$ и $d$. Поэтому самая общая форма конечного результата имеет следующий вид:

$$
\begin{aligned}
S_{N}(\{\mu\} \mid\{\lambda\})= & \sum_{\substack{\alpha \cup \bar{\alpha} \\
\gamma \cup \bar{\gamma}}} \prod_{j \in \gamma} a\left(\mu_{j}\right) \prod_{k \in \bar{\gamma}} d\left(\mu_{k}\right) \prod_{k \in \bar{\alpha}} a\left(\lambda_{k}\right) \prod_{j \in \alpha} d\left(\lambda_{j}\right) \\
& \times K_{N}\left(\{\mu\}_{\gamma},\{\mu\}_{\bar{\gamma}} \mid\{\lambda\}_{\alpha},\{\lambda\}_{\bar{\alpha}}\right) .
\end{aligned}
$$

Поясним обозначения, использованные в формуле (5.3). Набор параметров $\{\lambda\}$ разбит на два непересекающихся подмножества $\{\lambda\}=\{\lambda\}_{\alpha} \cup\{\lambda\}_{\bar{\alpha}}$. Аналогично $\{\mu\}=\{\mu\}_{\gamma} \cup\{\mu\}_{\bar{\gamma}}$. Данные разбиения независимы, за исключением условия $\#\{\lambda\}_{\alpha}=\#\{\mu\}_{\gamma}=n$, где $n=0,1, \ldots, N$. Разбиения параметров $\{\lambda\}$ и $\{\mu\}$ автоматически влекут два разбиения набора индексов $1, \ldots, N$ на $\{\alpha\} \cup\{\bar{\alpha}\}$ и $\{\gamma\} \cup\{\bar{\gamma}\}$. В каждом подмножестве параметры упорядочены естественным образом, например, $\left\{\lambda_{\alpha_{1}}, \lambda_{\alpha_{2}}, \ldots, \lambda_{\alpha_{n}}\right\}$, если $\alpha_{1}<\alpha_{2}<\cdots<\alpha_{n}$, и т. д. Сумма в формуле (5.3) берется по всем разбиениям указанного вида. Подобные обозначения будут использоваться ниже на протяжении всей статьи.

Символом $K_{N}\left(\{\mu\}_{\gamma},\{\mu\}_{\bar{\gamma}} \mid\{\lambda\}_{\alpha},\{\lambda\}_{\bar{\alpha}}\right)$ в формуле (5.3) обозначен коэффициент, возникающий при перестановке операторов, зависящий от $R$-матрицы и не зависящий от вакуумных собственных значений операторов $A$ и $D$. Наша цель найти явный вид этого коэффициента.

5.1. Выражение для общего коэффициента через старшие. Введем определение.

ОПРЕДЕЛЕНИЕ 5.1. Стариим коэфбициентом $K_{N}(\{\mu\} \mid\{\lambda\})$ называется коэффициент в формуле (5.3), соответствующий разбиению $\{\mu\}_{\bar{\gamma}}=\{\lambda\}_{\bar{\alpha}}=\varnothing$; сопряженным старшим коэффициентом $\bar{K}_{N}(\{\mu\} \mid\{\lambda\})$ называется коэффициент в формуле (5.3), соответствующий разбиению $\{\mu\}_{\gamma}=\{\lambda\}_{\alpha}=\varnothing$;

$$
\begin{aligned}
& K_{N}(\{\mu\} \mid\{\lambda\})=K_{N}\left(\{\mu\}_{\gamma}, \varnothing \mid\{\lambda\}_{\alpha}, \varnothing\right), \\
& \bar{K}_{N}(\{\mu\} \mid\{\lambda\})=K_{N}\left(\varnothing,\{\mu\}_{\bar{\gamma}} \mid \varnothing,\{\lambda\}_{\bar{\alpha}}\right) .
\end{aligned}
$$


Покажем, что произвольный коэффициент $K_{N}\left(\{\mu\}_{\gamma},\{\mu\}_{\bar{\gamma}} \mid\{\lambda\}_{\alpha},\{\lambda\}_{\bar{\alpha}}\right)$ в формуле (5.3) выражается через старший и сопряженный старший коэффициенты [28], [27]. Для этого зафиксируем некоторое разбиение $\{\mu\}=\{\mu\}_{\gamma} \cup\{\mu\}_{\bar{\gamma}}$ и $\{\lambda\}=\{\lambda\}_{\alpha} \cup\{\lambda\}_{\bar{\alpha}}$ и найдем коэффициент, соответствующий данному конкретному разбиению. Пользуясь (3.1), переупорядочим операторы $B$ и $C$ следующим образом:

$$
\left\langle 0\left|\prod_{j=1}^{N} C\left(\mu_{j}\right) \prod_{j=1}^{N} B\left(\lambda_{j}\right)\right| 0\right\rangle=\left\langle 0\left|\prod_{k \in \bar{\gamma}} C\left(\mu_{k}\right) \prod_{j \in \gamma} C\left(\mu_{j}\right) \cdot \prod_{j \in \alpha} B\left(\lambda_{j}\right) \prod_{k \in \bar{\alpha}} B\left(\lambda_{k}\right)\right| 0\right\rangle .
$$

Перепишем соотношение (3.10) в виде

$$
C(\mu) B(\lambda)=B(\lambda) C(\mu)+g(\lambda, \mu)(A(\mu) D(\lambda)-A(\lambda) D(\mu))
$$

и по аналогии с коммутационными соотношениями, рассмотренными в предыдущем разделе, будем называть слагаемые в правой части формулы (5.6) первой и второй схемой коммутации. Рассмотрим произвольный оператор $C\left(\mu_{s}\right)$ с аргументом $\mu_{s} \in\{\mu\}_{\gamma}$ и начнем двигать его направо, пользуясь соотношением (5.6). Предположим, что при коммутации с произведением $\prod_{j \in \alpha} B\left(\lambda_{j}\right)$ мы все время пользовались первой схемой. Тогда мы получим

$$
\prod_{j \in \alpha} B\left(\lambda_{j}\right) \cdot C\left(\mu_{s}\right) \cdot \prod_{k \in \bar{\alpha}} B\left(\lambda_{k}\right)|0\rangle .
$$

Ясно, что действие оператора $C\left(\mu_{s}\right)$ на вектор $\prod_{k \in \bar{\alpha}} B\left(\lambda_{k}\right)|0\rangle$ дает слагаемые, пропорциональные либо $a\left(\mu_{s}\right) d\left(\lambda_{\ell}\right)$, либо $a\left(\lambda_{\ell}\right) d\left(\mu_{s}\right)$, либо наконец $a\left(\lambda_{\ell}\right) d\left(\lambda_{\ell^{\prime}}\right)$, где $\lambda_{\ell}, \lambda_{\ell^{\prime}} \in\{\lambda\}_{\bar{\alpha}}$. Однако искомый коэффициент содержит функции $d(\mu)$ и $d(\lambda)$ только при $\mu \in\{\mu\}_{\bar{\gamma}}$ и $\lambda \in\{\lambda\}_{\alpha}$. Следовательно, состояние (5.7) не дает вклада в искомый коэффициент.

Мы приходим к выводу, что при коммутации любого из операторов $C\left(\mu_{s}\right)$ при $\mu_{s} \in\{\mu\}_{\gamma}$ с произведением $\prod_{j \in \alpha} B\left(\lambda_{j}\right)$ мы должны по крайней мере один раз воспользоваться второй схемой:

$$
\begin{aligned}
\prod_{j \in \gamma} C\left(\mu_{j}\right) & \cdot \prod_{j \in \alpha} B\left(\lambda_{j}\right)=\prod_{\substack{j \in \gamma \\
j \neq s}} C\left(\mu_{j}\right) \sum_{\ell \in \alpha} g\left(\lambda_{\alpha_{\ell}}, \mu_{s}\right) B\left(\lambda_{\alpha_{1}}\right) \cdots B\left(\lambda_{\alpha_{\ell-1}}\right) \\
& \times\left[A\left(\mu_{s}\right) D\left(\lambda_{\alpha_{\ell}}\right)-A\left(\lambda_{\alpha_{\ell}}\right) D\left(\mu_{s}\right)\right] B\left(\lambda_{\alpha_{\ell+1}}\right) \cdots B\left(\lambda_{\alpha_{n}}\right)+\mathscr{Z},
\end{aligned}
$$

где мы вновь обозначили символом $\mathscr{Z}$ все слагаемые, не дающие вклада в искомый коэффициент. Пользуясь теперь соотношениями (3.3), (3.7) и (3.4), мы перенесем операторы $A$ в крайнее левое положение, а операторы $D$ в крайнее правое. Повторяя эту процедуру для всех операторов $C(\mu)$ с $\mu \in\{\mu\}_{\gamma}$, мы в конечном итоге придем к формуле, аналогичной (5.3), с той лишь разницей, что вместо функций $a$ и $d$ мы получим операторы $A$ и $D$ :

$$
\begin{aligned}
\prod_{j \in \gamma} C\left(\mu_{j}\right) \cdot \prod_{j \in \alpha} B\left(\lambda_{j}\right)= & \sum_{\substack{\alpha+\cup \alpha_{-} \\
\gamma_{+} \cup \gamma_{-}}} \prod_{j \in \gamma_{+}} A\left(\mu_{j}\right) \prod_{k \in \alpha_{-}} A\left(\lambda_{k}\right) \prod_{j \in \alpha_{+}} D\left(\lambda_{j}\right) \prod_{k \in \gamma_{-}} D\left(\mu_{k}\right) \\
& \times K_{n}\left(\{\mu\}_{\gamma_{+}},\{\mu\}_{\gamma_{-}} \mid\{\lambda\}_{\alpha_{+}},\{\lambda\}_{\alpha_{-}}\right)+\mathscr{Z} .
\end{aligned}
$$


Здесь суммирование ведется по разбиениям набора $\{\lambda\}_{\alpha}$ на подмножества $\{\lambda\}_{\alpha}=\{\lambda\}_{\alpha_{+}} \cup\{\lambda\}_{\alpha_{-}}$и набора $\{\mu\}_{\gamma}$ на подмножества $\{\mu\}_{\gamma}=\{\mu\}_{\gamma_{+}} \cup\{\mu\}_{\gamma_{-}}$.

Допустим, что $\{\mu\}_{\gamma_{-}} \neq \varnothing$. Тогда при коммутации оператора $D\left(\mu_{s}\right)$ при $\mu_{s} \in\{\mu\}_{\gamma_{-}}$с произведением $\prod_{j \in \bar{\alpha}} B\left(\lambda_{j}\right)$ мы получим слагаемые, пропорциональные либо $d\left(\mu_{s}\right)$, либо $d\left(\lambda_{\ell}\right)$ с $\lambda_{\ell} \in\{\lambda\}_{\bar{\alpha}}$. Поскольку ни те, ни другие не должны присутствовать в конечном ответе, мы заключаем, что $\{\mu\}_{\gamma_{-}}=\varnothing$, а значит, и $\{\lambda\}_{\alpha_{-}}=\varnothing$. Следовательно,

$$
\prod_{j \in \gamma} C\left(\mu_{j}\right) \cdot \prod_{j \in \alpha} B\left(\lambda_{j}\right)=\prod_{j \in \gamma} A\left(\mu_{j}\right) \prod_{j \in \alpha} D\left(\lambda_{j}\right) \cdot K_{n}\left(\{\mu\}_{\gamma} \mid\{\lambda\}_{\alpha}\right)+\mathscr{Z}
$$

где $K_{n}\left(\{\mu\}_{\gamma} \mid\{\lambda\}_{\alpha}\right)$ - старший коэффициент, зависящий от наборов $\{\mu\}_{\gamma}$ и $\{\lambda\}_{\alpha}$. В итоге получаем

$$
\begin{aligned}
& \left\langle 0\left|\prod_{j=1}^{N} C\left(\mu_{j}\right) \prod_{j=1}^{N} B\left(\lambda_{j}\right)\right| 0\right\rangle \\
& =K_{n}\left(\{\mu\}_{\gamma} \mid\{\lambda\}_{\alpha}\right) \cdot\left\langle 0\left|\prod_{k \in \bar{\gamma}} C\left(\mu_{k}\right) \prod_{j \in \gamma} A\left(\mu_{j}\right) \cdot \prod_{j \in \alpha} D\left(\lambda_{j}\right) \prod_{k \in \bar{\alpha}} B\left(\lambda_{k}\right)\right| 0\right\rangle+\mathscr{Z} .
\end{aligned}
$$

Дальнейшие действия достаточно очевидны. Переместим все операторы $D$ в крайнее правое положение, а операторы $A$ в крайнее левое. При этом мы можем пользоваться лишь первой схемой коммутации (т. е. операторы $A$ и $D$ должны сохранять свои аргументы). В противном случае мы получим вклады, пропорциональные либо $a(\mu)$ при $\mu \in\{\mu\}_{\bar{\gamma}}$, либо $d(\lambda)$ при $\lambda \in\{\lambda\}_{\bar{\alpha}}$. Таким образом,

$$
\begin{aligned}
\langle 0| & \prod_{j=1}^{N} C\left(\mu_{j}\right) \prod_{j=1}^{N} B\left(\lambda_{j}\right)|0\rangle=K_{n}\left(\{\mu\}_{\gamma} \mid\{\lambda\}_{\alpha}\right) \cdot \prod_{j \in \gamma} a\left(\mu_{j}\right) \prod_{j \in \alpha} d\left(\lambda_{j}\right) \\
& \times \prod_{a \in \alpha} \prod_{b \in \bar{\alpha}} f\left(\lambda_{a}, \lambda_{b}\right) \prod_{a \in \gamma} \prod_{b \in \bar{\gamma}} f\left(\mu_{b}, \mu_{a}\right) \cdot\left\langle 0\left|\prod_{k \in \bar{\gamma}} C\left(\mu_{k}\right) \prod_{k \in \bar{\alpha}} B\left(\lambda_{k}\right)\right| 0\right\rangle+\mathscr{Z} .
\end{aligned}
$$

В оставшемся скалярном произведении, очевидно, нужный вклад дает лишь слагаемое, пропорциональное сопряженному старшему коэффициенту. В результате мы окончательно получаем

$$
\begin{aligned}
K_{N}\left(\{\mu\}_{\gamma},\{\mu\}_{\bar{\gamma}} \mid\{\lambda\}_{\alpha},\{\lambda\}_{\bar{\alpha}}\right)= & K_{n}\left(\{\mu\}_{\gamma} \mid\{\lambda\}_{\alpha}\right) \bar{K}_{N-n}\left(\{\mu\}_{\bar{\gamma}} \mid\{\lambda\}_{\bar{\alpha}}\right) \\
& \times \prod_{a \in \alpha} \prod_{b \in \bar{\alpha}} f\left(\lambda_{a}, \lambda_{b}\right) \prod_{a \in \gamma} \prod_{b \in \bar{\gamma}} f\left(\mu_{b}, \mu_{a}\right) .
\end{aligned}
$$

5.2. Старший коэффициент. Получим теперь явную формулу для старшего коэффициента $K_{N}$. Для этого мы должны в формуле (5.3) выделить единственное слагаемое, соответствующее разбиению $\{\mu\}_{\gamma}=\{\mu\},\{\lambda\}_{\alpha}=\{\lambda\}$. 
Рассмотрим действие оператора $C\left(\mu_{N}\right)$ на вектор $\prod_{j=1}^{N} B\left(\lambda_{j}\right)|0\rangle$. Воспользовавшись формулой (3.19), мы получаем

$$
\begin{aligned}
& C\left(\mu_{N}\right) \prod_{j=1}^{N} B\left(\lambda_{j}\right)|0\rangle \\
& \left.\quad=\sum_{\ell=1}^{N} a\left(\mu_{N}\right) d\left(\lambda_{\ell}\right) g\left(\lambda_{\ell}, \mu_{N}\right) \prod_{\substack{a=1 \\
a \neq \ell}}^{N} f\left(\lambda_{a}, \mu_{N}\right) f\left(\lambda_{\ell}, \lambda_{a}\right)\right) \cdot \prod_{\substack{j=1 \\
j \neq \ell}}^{N} B\left(\lambda_{j}\right)|0\rangle+\mathscr{Z} .
\end{aligned}
$$

Домножая равенство (5.14) на дуальный вектор $\langle 0| \prod_{j=1}^{N-1} C\left(\mu_{j}\right)$, мы немедленно приходим к рекуррентному соотношению для старшего коэффициента

$$
\begin{aligned}
& K_{N}(\{\mu\} \mid\{\lambda\}) \\
& \quad=\sum_{\ell=1}^{N} g\left(\lambda_{\ell}, \mu_{N}\right) \prod_{\substack{a=1 \\
a \neq \ell}}^{N}\left(f\left(\lambda_{a}, \mu_{N}\right) f\left(\lambda_{\ell}, \lambda_{a}\right)\right) \cdot K_{N-1}\left(\left\{\mu \neq \mu_{N}\right\} \mid\left\{\lambda \neq \lambda_{\ell}\right\}\right) .
\end{aligned}
$$

Соотношение (5.15) вместе с начальным условием

$$
K_{1}(\mu \mid \lambda)=g(\lambda, \mu)
$$

следующим из (5.2), однозначно фиксируют старший коэффициент и позволяют вычислять его рекуррентным образом. Существует, однако, простая явная формула для старшего коэффициента при любом $N$ [30].

ПреДЛОжеНИЕ 5.1. Явный вид старшего коэффичиента $K_{N}(\{\mu\} \mid\{\lambda\}) \partial а$ ется формулой

$$
K_{N}(\{\mu\} \mid\{\lambda\})=\prod_{a, b=1}^{N} h\left(\lambda_{a}, \mu_{b}\right) \prod_{a>b}^{N} g\left(\lambda_{a}, \lambda_{b}\right) g\left(\mu_{b}, \mu_{a}\right) \cdot \operatorname{det}_{N} t\left(\lambda_{j}, \mu_{k}\right),
$$

где функиия $t(\lambda, \mu)$ определена в формулах (2.12)-(2.14).

Для доказательства предложения 5.1 нам потребуется вспомогательная лемма.

Лемма 5.1. Рассмотрим случай тригонометрической $R$-матрицы. Пусть

$$
u_{k}=\frac{\prod_{a=1}^{N} \operatorname{sh}\left(\lambda_{a}-\mu_{k}\right)}{\prod_{a=1, a \neq k}^{N} \operatorname{sh}\left(\mu_{a}-\mu_{k}\right)} .
$$

Тогда

$$
\sum_{k=1}^{N} u_{k} t\left(\lambda_{j}, \mu_{k}\right)=\prod_{a=1}^{N} \frac{\operatorname{sh}\left(\lambda_{j}-\lambda_{a}+\eta\right)}{\operatorname{sh}\left(\lambda_{j}-\mu_{a}+\eta\right)} .
$$

ДОкАЗАТЕЛЬСТво ЛЕммЫ 5.1. Пусть

$$
\sum_{k=1}^{N} u_{k} t\left(\lambda_{j}, \mu_{k}\right)=G_{j}
$$


Рассмотрим вспомогательный интеграл

$$
I=\frac{1}{2 \pi i} \int_{\Gamma} \frac{\operatorname{sh} \eta d z}{\operatorname{sh}\left(\lambda_{j}-z\right) \operatorname{sh}\left(\lambda_{j}-z+\eta\right)} \prod_{a=1}^{N} \frac{\operatorname{sh}\left(\lambda_{a}-z\right)}{\operatorname{sh}\left(\mu_{a}-z\right)} .
$$

Контур интегрирования Г представляет собой границу горизонтальной полосы шириной $i \pi$. Поскольку подынтегральная функция в (5.21) периодична с периодом $i \pi$ и экспоненциально убывает при $z \rightarrow \pm \infty$, мы заключаем, что $I=0$. С другой стороны, интеграл (5.21) равен сумме вычетов внутри контура. Сумма вычетов в точках $z=\mu_{a}$ равна $-G_{j}$. Кроме полюсов при $z=\mu_{a}$ подынтегральное выражение содержит еще один простой полюс в точке $z=\lambda_{j}+\eta$. Приравнивая нулю общую сумму вычетов, мы приходим к равенству

$$
G_{j}=\frac{\operatorname{sh}\left(\lambda_{j}-\lambda_{a}+\eta\right)}{\operatorname{sh}\left(\lambda_{j}-\mu_{a}+\eta\right)}
$$

что и требовалось доказать.

ЗАмЕчАниЕ. Приведенное доказательство очевидном образом переносится на случай рациональной $R$-матрицы. При этом в качестве контура интегрирования $\Gamma$ в интеграле (5.21) следует брать окружность вокруг бесконечно удаленной точки.

ДОКАЗАТЕЛЬСТВО ПРЕДЛОЖЕНИЯ 5.1. Прибавим к последнему столбцу матрицы $t\left(\lambda_{j}, \mu_{k}\right)$ все остальные столбцы, умноженные на коэффициенты $u_{k} / u_{N}$. Тогда в силу леммы 5.1 последний столбец оказывается равным $G_{j} / u_{N}$. Раскладывая затем детерминант по последнему столбцу, мы приходим к рекуррентному соотношению

$$
\operatorname{det}_{N} t\left(\lambda_{j}, \mu_{k}\right)=\left.\frac{1}{u_{N}} \sum_{\ell=1}^{N}(-1)^{N+\ell} \prod_{a=1}^{N} \frac{h\left(\lambda_{\ell}, \lambda_{a}\right)}{h\left(\lambda_{\ell}, \mu_{a}\right)} \cdot \operatorname{det}_{N-1} t\left(\lambda_{j}, \mu_{k}\right)\right|_{\substack{j \neq \ell \\ k \neq N}} .
$$

Подставляя это соотношение в формулу (5.17), мы видим, что функция $K_{N}$, определяемая этим равенством, действительно удовлетворяет рекуррентному соотношению (5.15). Остается проверить, что при $N=1$ формула (5.17) дает $g(\lambda, \mu)$. Предложение 5.1 доказано.

ЗАмЕчАНИЕ. В первом доказательстве формулы (5.17) (см. [30]) существенным образом использовалась связь между старшим коэффициентом и статистической суммой шестивершинной модели со специальными граничными условиями. Доказательство, приведенное выше, является прямым и основано исключительно на коммутационных соотношениях в алгебре (2.2).

Легко видеть, что сопряженный старший коэффициент также дается формулой (5.17), в которой следует заменить параметры $\{\lambda\}$ на параметры $\{\mu\}$ :

$$
\bar{K}_{N}(\{\mu\} \mid\{\lambda\})=\prod_{a, b=1}^{N} h\left(\mu_{b}, \lambda_{a}\right) \prod_{a>b}^{N} g\left(\lambda_{a}, \lambda_{b}\right) g\left(\mu_{b}, \mu_{a}\right) \cdot \operatorname{det}_{N} t\left(\mu_{k}, \lambda_{j}\right) .
$$


В результате мы получаем окончательный ответ для скалярного произведения:

$$
\begin{aligned}
S_{N}(\{\mu\} \mid\{\lambda\}) \\
=\prod_{a>b}^{N} g\left(\lambda_{a}, \lambda_{b}\right) g\left(\mu_{b}, \mu_{a}\right) \sum_{\substack{\alpha \cup \bar{\alpha} \\
\gamma \cup \bar{\gamma}}}(-1)^{P_{\alpha}+P_{\gamma}} \prod_{j \in \gamma} a\left(\mu_{j}\right) \prod_{k \in \bar{\gamma}} d\left(\mu_{k}\right) \prod_{k \in \bar{\alpha}} a\left(\lambda_{k}\right) \prod_{j \in \alpha} d\left(\lambda_{j}\right) \\
\quad \times \operatorname{det}_{\substack{j \in \alpha \\
k \in \gamma}} t\left(\lambda_{j}, \mu_{k}\right) \operatorname{det}_{\substack{j \in \bar{\alpha} \\
k \in \bar{\gamma}}} t\left(\mu_{k}, \lambda_{j}\right) \\
\quad \times \prod_{a \in \alpha} \prod_{b \in \gamma} h\left(\lambda_{a}, \mu_{b}\right) \prod_{a \in \bar{\alpha}} \prod_{b \in \bar{\gamma}} h\left(\mu_{b}, \lambda_{a}\right) \prod_{a \in \alpha} \prod_{b \in \bar{\alpha}} h\left(\lambda_{a}, \lambda_{b}\right) \prod_{a \in \gamma} \prod_{b \in \bar{\gamma}} h\left(\mu_{b}, \mu_{a}\right) .
\end{aligned}
$$

Здесь символами $P_{\alpha}$ и $P_{\gamma}$ обозначены четности перестановок

$$
\begin{aligned}
P\left(\alpha_{1}, \ldots, \alpha_{n}, \bar{\alpha}_{1}, \ldots, \bar{\alpha}_{N-n}\right) & =1, \ldots, N \\
P\left(\gamma_{1}, \ldots, \gamma_{n}, \bar{\gamma}_{1}, \ldots, \bar{\gamma}_{N-n}\right) & =1, \ldots, N .
\end{aligned}
$$

\section{6. Частные случаи скалярного произведения}

Структура выражения (5.25) напоминает формулу Лапласа для детерминанта суммы двух матриц. Действительно, если нам даны две функции $U(\lambda, \mu)$ и $V(\lambda, \mu)$, то

$$
\operatorname{det}_{N}\left(U\left(\lambda_{j}, \mu_{k}\right)+V\left(\lambda_{j}, \mu_{k}\right)\right)=\sum_{\substack{\alpha \cup \bar{\alpha} \\ \gamma \cup \bar{\gamma}}}(-1)^{P_{\alpha}+P_{\gamma}} \operatorname{det}_{\substack{j \in \alpha \\ k \in \gamma}} U\left(\lambda_{j}, \mu_{k}\right) \operatorname{det}_{\substack{j \in \bar{\alpha} \\ k \in \bar{\gamma}}} V\left(\lambda_{j}, \mu_{k}\right) .
$$

Однако наличие в формуле (5.25) двойных произведений функций $h$ мешает переписать ее в более компактном виде и свести к детерминанту суммы двух матриц. Пользоваться же в конкретных задачах непосредственно формулой (5.25), представляющей собой хоть и явный, но достаточно громоздкий ответ, крайне неудобно.

K счастью, в приложениях, особенно при вычислении корреляционных функций, наиболее общий вид скалярного произведения оказывается, как правило, не нужен. Гораздо чаще приходится иметь дело с ситуацией, когда один из наборов, например параметры $\{\mu\}$, являются произвольными комплексными числами, в то время как параметры $\{\lambda\}$ удовлетворяют уравнениям Бете (4.7). Рассмотрим следующий пример. Пусть требуется вычислить матричный элемент некоторого оператора $\mathscr{O}$,

$$
\langle\mathscr{O}\rangle=\left\langle 0\left|\prod_{j=1}^{N_{1}} C\left(\mu_{j}\right) \cdot \mathscr{O} \cdot \prod_{j=1}^{N_{2}} B\left(\lambda_{j}\right)\right| 0\right\rangle,
$$

между двумя физическими состояниями. Это означает, что оба набора $\{\mu\}$ и $\{\lambda\}$ являются решениями уравнений Бете. Допустим, что нам удалось вычислить действие оператора $\mathscr{O}$, например, на дуальное состояние $\langle 0| \prod_{j=1}^{N_{1}} C\left(\mu_{j}\right)$ в виде некоторой линейной комбинации новых дуальных векторов:

$$
\langle 0| \prod_{j=1}^{N_{1}} C\left(\mu_{j}\right) \cdot \mathscr{O}=\sum_{\ell} \theta_{\ell}\langle 0| \prod_{j=1}^{N_{\ell}} C\left(\nu_{j}^{(\ell)}\right) .
$$


Новые аргументы $\nu_{j}^{(\ell)}$ операторов $C$, вообще говоря, уже не удовлетворяют никаким дополнительным условиям. Однако вектор $\prod_{j=1}^{N_{2}} B\left(\lambda_{j}\right)|0\rangle$ по-прежнему является собственным вектором трансферматрицы, и вычисление матричного элемента сводится к вычислению скалярных произведений, в которых набор параметров $\{\lambda\}$ является решением уравнений Бете. $\mathrm{K}$ изучению таких скалярных произведений мы и переходим.

6.1. Вспомогательное тождество. Сначала докажем лемму, играющую важную роль в дальнейших вычислениях. Рассмотрим вспомогательную функцию $G_{N}^{(n)}$, зависящую при фиксированных $n$ и $N(0 \leqslant n \leqslant N)$ от трех наборов комплексных переменных $\left\{\xi_{1}, \ldots, \xi_{n}\right\},\left\{\nu_{1}, \ldots, \nu_{N-n}\right\}$ и $\left\{\lambda_{1}, \ldots, \lambda_{N}\right\}$ :

$$
\begin{aligned}
& G_{N}^{(n)}(\{\xi\},\{\nu\},\{\lambda\})=\sum_{\substack{\alpha \cup \bar{\alpha} \\
\#\{\alpha\}=n}}(-1)^{P_{\alpha}} \operatorname{det}_{\substack{j \in \alpha \\
k=1, \ldots, n}} t\left(\lambda_{j}, \xi_{k}\right) \cdot \operatorname{det}_{\substack{j \in \bar{\alpha} \\
k=1, \ldots, N-n}} t\left(\nu_{k}, \lambda_{j}\right) \\
& \times\left\{\prod_{a=1}^{n} \prod_{b=1}^{N-n} h\left(\nu_{b}, \xi_{a}\right) \prod_{a \in \alpha} \prod_{b \in \bar{\alpha}} h\left(\lambda_{b}, \lambda_{a}\right)-\prod_{a=1}^{n} \prod_{b \in \bar{\alpha}} h\left(\lambda_{b}, \xi_{a}\right) \prod_{a=1}^{N-n} \prod_{b \in \alpha} h\left(\nu_{a}, \lambda_{b}\right)\right\} \\
& \times \prod_{a=1}^{n} \prod_{b \in \alpha} h\left(\lambda_{b}, \xi_{a}\right) \prod_{a=1}^{N-n} \prod_{b \in \bar{\alpha}} h\left(\nu_{a}, \lambda_{b}\right) .
\end{aligned}
$$

Здесь суммирование ведется по всем разбиениям набора $\{\lambda\}$ на подмножества

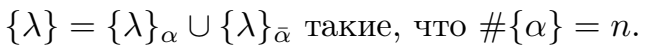

ЛЕмма 6.1. Для произвольных комплексных чисел $\{\xi\},\{\nu\} u\{\lambda\}$

$$
G_{N}^{(n)}(\{\xi\},\{\nu\},\{\lambda\}) \equiv 0 .
$$

ДокАЗАтельство. При $N=1$ утверждение леммы очевидно, поскольку либо $n=0$, либо $N-n=0$. Пусть $G_{N-1}^{(n)}(\{\xi\},\{\nu\},\{\lambda\})=0$. Рассмотрим свойства $G_{N}^{(n)}$ как функции переменных $\lambda_{1}, \ldots, \lambda_{N}$ при фиксированных остальных параметрах. Очевидно, что $G_{N}^{(n)}=0$ при $\lambda_{j}=\lambda_{k}(j, k=1, \ldots, N, j \neq k)$. Следовательно, комбинация

$$
\widetilde{G}_{N}^{(n)}(\{\xi\},\{\nu\},\{\lambda\})=\prod_{k<j} g\left(\lambda_{j}, \lambda_{k}\right) \cdot G_{N}^{(n)}(\{\xi\},\{\nu\},\{\lambda\})
$$

не имеет полюсов при $\lambda_{j}=\lambda_{k}$. Поэтому единственными возможными сингулярностями функции $\widetilde{G}_{N}^{(n)}$ являются полюсы в точках $\lambda_{j}=\xi_{k}$ и $\lambda_{j}=\nu_{k}$. Легко видеть, что вычеты в этих полюсах пропорциональны $\widetilde{G}_{N-1}^{(n)}$, например,

$$
\begin{aligned}
& \left.\operatorname{Res} \widetilde{G}_{N}^{(n)}(\{\xi\},\{\nu\},\{\lambda\})\right|_{\lambda_{1}=\xi_{1}}=\widetilde{G}_{N-1}^{(n)}\left(\left\{\xi \neq \xi_{1}\right\},\{\nu\},\left\{\lambda \neq \lambda_{1}\right\}\right) \\
& \quad \times \operatorname{sh} \eta \prod_{b=2}^{N} f\left(\lambda_{b}, \xi_{1}\right) \prod_{a=1}^{n} h\left(\xi_{1}, \xi_{a}\right) \prod_{a=1}^{N-n} h\left(\nu_{a}, \xi_{1}\right) .
\end{aligned}
$$

В силу индуктивного предположения эти вычеты раны нулю. Таким образом, $\widetilde{G}_{N}^{(n)}$ является голоморфной функцией переменных $\lambda_{j}$ во всей комплексной плоскости. Остается заметить, что $\widetilde{G}_{N}^{(n)}(\{\xi\},\{\nu\},\{\lambda\})$ убывает при $\left|\operatorname{Re} \lambda_{j}\right| \rightarrow \infty$. Следовательно, $G_{N}^{(n)}(\{\xi\},\{\nu\},\{\lambda\})=0$, что и требовалось доказать. 


\section{2. Скалярное произведение собственного состояния на произ-} вольное. Рассмотрим теперь скалярное произведение в случае, когда одно из состояний является собственным вектором твистованной трансферматрицы ${ }^{1}$. Пусть, например, в состоянии $\prod_{j=1}^{N} B\left(\lambda_{j}\right)|0\rangle$ параметры $\{\lambda\}$ удовлетворяют уравнениям (4.7). Тогда мы можем выразить функцию $a\left(\lambda_{j}\right)$ через $d\left(\lambda_{j}\right)$ :

$$
a\left(\lambda_{j}\right)=\varkappa d\left(\lambda_{j}\right)(-1)^{N-1} \prod_{a=1}^{N} \frac{h\left(\lambda_{j}, \lambda_{a}\right)}{h\left(\lambda_{a}, \lambda_{j}\right)} .
$$

Подставляя это в равенство (5.25), получаем

$$
\begin{aligned}
& \left\langle 0\left|\prod_{j=1}^{N} C\left(\mu_{j}\right)\right| \psi_{\varkappa}(\{\lambda\})\right\rangle=\prod_{a>b}^{N} g\left(\lambda_{a}, \lambda_{b}\right) g\left(\mu_{b}, \mu_{a}\right) \cdot \prod_{j=1}^{N} d\left(\lambda_{j}\right) \\
& \times \sum_{\substack{\alpha \cup \bar{\alpha} \\
\gamma \cup \bar{\gamma}}}(-1)^{P_{\alpha}+P_{\gamma}}(-1)^{n N-n} \varkappa^{N-n} \prod_{a \in \gamma} a\left(\mu_{a}\right) \prod_{b \in \bar{\gamma}} d\left(\mu_{b}\right) \cdot \operatorname{det}_{\substack{j \in \alpha \\
k \in \gamma}} t\left(\lambda_{j}, \mu_{k}\right) \operatorname{det}_{\substack{j \in \bar{\alpha} \\
k \in \bar{\gamma}}} t\left(\mu_{k}, \lambda_{j}\right) \\
& \times \prod_{a \in \gamma} \prod_{b \in \alpha} h\left(\lambda_{b}, \mu_{a}\right) \prod_{a \in \bar{\alpha}} \prod_{b \in \bar{\gamma}} h\left(\mu_{b}, \lambda_{a}\right) \prod_{a \in \bar{\alpha}} \prod_{b \in \alpha} h\left(\lambda_{a}, \lambda_{b}\right) \prod_{a \in \gamma} \prod_{b \in \bar{\gamma}} h\left(\mu_{b}, \mu_{a}\right),
\end{aligned}
$$

где $n=\#\{\alpha\}$. Для каждого конкретного разбиения $\{\gamma\} \cup\{\bar{\gamma}\}$ можно положить $\{\mu\}_{\gamma}=\left\{\xi_{1}, \ldots, \xi_{n}\right\},\{\mu\}_{\bar{\gamma}}=\left\{\nu_{1}, \ldots, \nu_{N-n}\right\}$ и воспользоваться леммой 6.1. Тогда

$$
\begin{aligned}
\langle 0| & \prod_{j=1}^{N} C\left(\mu_{j}\right)\left|\psi_{\varkappa}(\{\lambda\})\right\rangle=\prod_{a>b}^{N} g\left(\lambda_{a}, \lambda_{b}\right) g\left(\mu_{b}, \mu_{a}\right) \cdot \prod_{j=1}^{N} d\left(\lambda_{j}\right) \\
& \times \sum_{\substack{\alpha \cup \bar{\alpha} \\
\gamma \cup \bar{\gamma}}}(-1)^{P_{\alpha}+P_{\gamma}}(-1)^{n N-n} \varkappa^{N-n} \prod_{a \in \gamma} a\left(\mu_{a}\right) \prod_{\substack{ \\
b \in \bar{\gamma}}} d\left(\mu_{b}\right) \cdot \operatorname{det}_{\substack{j \in \alpha \\
k \in \gamma}} t\left(\lambda_{j}, \mu_{k}\right) \operatorname{det}_{\substack{j \in \bar{\alpha} \\
k \in \bar{\gamma}}} t\left(\mu_{k}, \lambda_{j}\right) \\
& \times \prod_{a \in \gamma} \prod_{b=1}^{N} h\left(\lambda_{b}, \mu_{a}\right) \prod_{a=1}^{N} \prod_{b \in \bar{\gamma}} h\left(\mu_{b}, \lambda_{a}\right) .
\end{aligned}
$$

Теперь уже получившаяся сумма по разбиениям сводится к формуле Лапласа. Действительно, полагая в (6.1)

$$
\begin{aligned}
& U\left(\lambda_{j}, \mu_{k}\right)=(-1)^{N-1} a\left(\mu_{k}\right) t\left(\lambda_{j}, \mu_{k}\right) \prod_{a=1}^{N} h\left(\lambda_{a}, \mu_{k}\right), \\
& V\left(\lambda_{j}, \mu_{k}\right)=\varkappa d\left(\mu_{k}\right) t\left(\mu_{k}, \lambda_{j}\right) \prod_{a=1}^{N} h\left(\mu_{k}, \lambda_{a}\right),
\end{aligned}
$$

мы видим, что (6.1) в точности воспроизводит равенство (6.10). Таким образом мы приходим к следующему утверждению [38].

ПРЕДЛОЖЕНИЕ 6.1. Пусть набор параметров $\{\lambda\}$ удовлетворяет системе твистованных уравнений Бете (4.7), а параметры $\{\mu\}$ суть произвольные

\footnotetext{
${ }^{1}$ Все приведенные ниже рассуждения остаются справедливы и для обычной трансферматрицы. В этом случае следует просто положить в формулах $\varkappa=1$.
} 
комплексные числа. Тогда

$$
\left\langle 0\left|\prod_{j=1}^{N} C\left(\mu_{j}\right)\right| \psi_{\varkappa}(\{\lambda\})\right\rangle=\prod_{a>b}^{N} g\left(\lambda_{a}, \lambda_{b}\right) g\left(\mu_{b}, \mu_{a}\right) \cdot \prod_{j=1}^{N} d\left(\lambda_{j}\right) \cdot \operatorname{det}_{N} \Omega_{\varkappa}\left(\lambda_{j}, \mu_{k}\right),
$$

¿əe

$$
\Omega_{\varkappa}\left(\lambda_{j}, \mu_{k}\right)=a\left(\mu_{k}\right) t\left(\lambda_{j}, \mu_{k}\right) \prod_{a=1}^{N} h\left(\lambda_{a}, \mu_{k}\right)-(-1)^{N} \varkappa d\left(\mu_{k}\right) t\left(\mu_{k}, \lambda_{j}\right) \prod_{a=1}^{N} h\left(\mu_{k}, \lambda_{a}\right) .
$$

Для случая $X X Z$-цепочки Гейзенберга формула (6.12) была доказана иным способом, с помощью перехода к твистованному представлению для матрицы монодромии [25]. Доказательство, наиболее близкое к приведенному выше, было дано в работе [39].

Аналогично может быть рассмотрен случай, когда собственным состоянием твистованной трансферматрицы является дуальный вектор. При этом формула (6.12) не изменяется:

$$
\left\langle\psi_{\varkappa}(\{\lambda\})\left|\prod_{j=1}^{N} B\left(\mu_{j}\right)\right| 0\right\rangle=\prod_{a>b}^{N} g\left(\lambda_{a}, \lambda_{b}\right) g\left(\mu_{b}, \mu_{a}\right) \cdot \prod_{j=1}^{N} d\left(\lambda_{j}\right) \cdot \operatorname{det}_{N} \Omega_{\varkappa}\left(\lambda_{j}, \mu_{k}\right) .
$$

Матрица $\Omega_{\varkappa}\left(\lambda_{j}, \mu_{k}\right)$ оказывается тесно связанной с собственными значениями твистованной трансферматрицы (4.11). Непосредственным вычислением легко проверить [25], что

$$
\Omega_{\varkappa}\left(\lambda_{j}, \mu_{k}\right)=\operatorname{sh} \eta \cdot \prod_{a=1}^{N} g^{-1}\left(\lambda_{a}, \mu_{k}\right) \cdot \frac{\partial \tau_{\varkappa}\left(\mu_{k} \mid\{\lambda\}\right)}{\partial \lambda_{j}} .
$$

Таким образом, формулы (6.12), (6.14) могут быть переписаны в виде

$$
\begin{aligned}
& \left\langle\psi_{\varkappa}(\{\lambda\})\left|\prod_{j=1}^{N} B\left(\mu_{j}\right)\right| 0\right\rangle=\left\langle 0\left|\prod_{j=1}^{N} C\left(\mu_{j}\right)\right| \psi_{\varkappa}(\{\lambda\})\right\rangle \\
& \quad=\prod_{j=1}^{N} d\left(\lambda_{j}\right) \cdot \mathscr{X}_{N}^{-1}(\{\mu\},\{\lambda\}) \cdot \operatorname{det}_{N}\left(\frac{\partial}{\partial \lambda_{j}} \tau_{\varkappa}\left(\mu_{k} \mid\{\lambda\}\right)\right),
\end{aligned}
$$

где $\mathscr{X}_{N}(\{\mu\},\{\lambda\})$ - детерминант Коши:

$$
\mathscr{X}_{N}(\{\mu\},\{\lambda\})=\operatorname{det}_{N}\left(\frac{1}{\operatorname{sh}\left(\mu_{k}-\lambda_{j}\right)}\right)=\frac{\prod_{a>b}^{N} \operatorname{sh}\left(\lambda_{a}-\lambda_{b}\right) \operatorname{sh}\left(\mu_{b}-\mu_{a}\right)}{\prod_{a, b=1}^{N} \operatorname{sh}\left(\mu_{b}-\lambda_{a}\right)} .
$$

\section{3. Ортогональность собственных функций.}

ПреДЛОЖЕНИЕ 6.2. Пусть наборы $\{\lambda\} u\{\mu\}$ суть два различных решения твистованных уравнений Бете (4.7). Тогда

$$
\left\langle\psi_{\varkappa}(\{\mu\}) \mid \psi_{\varkappa}(\{\lambda\})\right\rangle=0 .
$$


ДокАЗАТЕЛЬСтво [37]. Предположим, что $\mu_{k} \neq \lambda_{j}, j, k=1, \ldots, N$. С помощью уравнений Бете выразим в матрице $\Omega_{\varkappa}$ функции $d\left(\mu_{k}\right)$ через $a\left(\mu_{k}\right)$. Тогда матрица $\Omega_{\varkappa}$ приобретает вид

$$
\Omega_{\varkappa}\left(\lambda_{j}, \mu_{k}\right)=a\left(\mu_{k}\right) \prod_{a=1}^{N} h\left(\lambda_{a}, \mu_{k}\right)\left[t\left(\lambda_{j}, \mu_{k}\right)+t\left(\mu_{k}, \lambda_{j}\right) \prod_{a=1}^{N} \frac{h\left(\mu_{k}, \lambda_{a}\right) h\left(\mu_{a}, \mu_{k}\right)}{h\left(\lambda_{a}, \mu_{k}\right) h\left(\mu_{k}, \mu_{a}\right)}\right] .
$$

Легко видеть, что получившаяся матрица имеет собственный вектор

$$
v_{j}=\frac{\prod_{a=1, a \neq j}^{N} g\left(\lambda_{a}, \lambda_{j}\right)}{\prod_{a=1}^{N} g\left(\mu_{a}, \lambda_{j}\right)}
$$

с нулевым собственным значением. Доказательство тождества

$$
\sum_{j=1}^{N} \Omega_{\varkappa}\left(\lambda_{j}, \mu_{k}\right) v_{j}=0
$$

вполне аналогично доказательству леммы 5.1.

Если некоторые переменные $\mu_{k}$ совпадают с переменными $\lambda_{k}$, то сначала следует перейти к пределу $\mu_{k} \rightarrow \lambda_{k}$ в соответствующих столбцах матрицы (6.13), после чего уже выразить функции $d\left(\mu_{k}\right)$ через $a\left(\mu_{k}\right)$. Легко видеть, что и в этом случае получившаяся матрица имеет тот же самый собственный вектор (6.20) с нулевым собственным значением. Предложение 6.2 доказано.

При $\{\mu\}=\{\lambda\}$ мы получаем формулу для квадрата нормы собственной функции твистованной трансферматрицы ${ }^{2}$ [28]. Отметим, что если все $\mu_{k}=\lambda_{k}$, $k=1, \ldots, N$, то все компоненты вектора $v_{j}$ обращаются в нуль и приведенное выше доказательство ортогональности, разумеется, теряет силу. Путем несложных вычислений находим

$$
\left\langle\psi_{\varkappa}(\{\lambda\}) \mid \psi_{\varkappa}(\{\lambda\})\right\rangle=\prod_{j=1}^{N} d\left(\lambda_{j}\right) \prod_{\substack{a, b=1 \\ a \neq b}}^{N} g\left(\lambda_{a}, \lambda_{b}\right) \cdot \operatorname{det}_{N}\left(-\operatorname{sh} \eta \frac{\partial}{\partial \lambda_{k}} \mathscr{Y}_{\varkappa}\left(\lambda_{j} \mid\{\lambda\}\right)\right) .
$$

В дальнейшем при вычислении корреляционных функций в термодинамическом пределе нам потребуется представление для квадрата нормы собственной функции в более детальном виде. Используя представление (4.5) для $\mathscr{Y}_{\varkappa}(\mu \mid\{\lambda\})$, несложно получить

$$
\operatorname{det}_{N}\left(\operatorname{sh} \eta \frac{\partial}{\partial \lambda_{k}} \mathscr{Y}_{\varkappa}\left(\lambda_{j} \mid\{\lambda\}\right)\right)=\prod_{j=1}^{N}\left(a\left(\lambda_{j}\right) \hat{\rho}\left(\lambda_{j}\right)\right) \prod_{j, k=1}^{N} h\left(\lambda_{j}, \lambda_{k}\right) \operatorname{det}_{N}\left(\delta_{j k}-\frac{K\left(\lambda_{j}-\lambda_{k}\right)}{\hat{\rho}\left(\lambda_{k}\right)}\right)
$$

\footnotetext{
${ }^{2}$ Мы используем устоявшийся термин "квадрат нормы”, хотя, вообще говоря, дуальная собственная функция $\left\langle\psi_{\varkappa}(\{\lambda\})\right|$ не является эрмитово сопряженной к собственной функции $\left|\psi_{\varkappa}(\{\lambda\})\right\rangle$. Однако в моделях, описывающих физические системы, свойство эрмитовой сопряженности восстанавливается при $\varkappa=1$.
} 
где

$$
\hat{\rho}(\lambda)=\frac{d}{d \lambda} \log \frac{a(\lambda)}{d(\lambda)}+\sum_{k=1}^{N} K\left(\lambda-\lambda_{k}\right)
$$

И

$$
K(\lambda)= \begin{cases}\frac{\operatorname{sh} 2 \eta}{\operatorname{sh}(\lambda-\eta) \operatorname{sh}(\lambda+\eta)} & \text { для тригонометрической } R \text {-матрицы, } \\ \frac{2 \eta}{(\lambda-\eta)(\lambda+\eta)} & \text { для рациональной } R \text {-матрицы. }\end{cases}
$$

6.4. Тождество для детерминантов. Рассмотрим скалярное произведение собственной функции твистованной трансферматрицы $\mathscr{T}_{\varkappa}(\mu)$ на собственную функцию обычной трансферматрицы $\mathscr{T}(\mu)$ :

$$
\left\langle\psi_{\varkappa}(\{\mu\}) \mid \psi(\{\lambda\})\right\rangle .
$$

Данное скалярное произведение может быть вычислено двумя способами. Вопервых, мы можем считать состояние $|\psi(\{\lambda\})\rangle$ частным случаем произвольного вектора и воспользоваться формулой (6.14), заменив в ней параметры $\mu$ на $\lambda$. Во-вторых, мы можем считать состояние $\left\langle\psi_{\varkappa}(\{\mu\})\right|$ частным случаем произвольного вектора и воспользоваться формулой (6.12), положив в ней $\varkappa=1$. Поскольку ответы, полученные обоими способами, должны совпадать, мы приходим к соотношению

$$
\prod_{j=1}^{N} d\left(\lambda_{j}\right) \cdot \operatorname{det}_{N}\left(\frac{\partial}{\partial \lambda_{j}} \tau_{\varkappa}\left(\mu_{k} \mid\{\lambda\}\right)\right)=\prod_{j=1}^{N} d\left(\mu_{j}\right) \cdot \operatorname{det}_{N}\left(-\frac{\partial}{\partial \mu_{j}} \tau\left(\lambda_{k} \mid\{\mu\}\right)\right)
$$

или, в терминах матрицы $\Omega_{\varkappa}$,

$$
\prod_{j=1}^{N} d\left(\lambda_{j}\right) \cdot \operatorname{det}_{N} \Omega_{\varkappa}\left(\mu_{k}, \lambda_{j}\right)=\prod_{j=1}^{N} d\left(\mu_{j}\right) \cdot \operatorname{det}_{N} \Omega\left(\lambda_{k}, \mu_{j}\right), \quad \Omega=\left.\Omega_{\varkappa}\right|_{\varkappa=1} .
$$

Подчеркнем, что равенства (6.27), (6.28) не являются тождествами, а выполняются только лишь при условии, что параметры $\{\mu\}$ являются решением твистованных уравнений Бете (4.7), а параметры $\{\lambda\}$ удовлетворяют обычным уравнениям Бете (4.6). Однако если исключить из этих равенств функции $a$ и $d$, то легко получить важное тождество для детерминантов, используемое при вычислении корреляционных функций.

Пользуясь уравнениями (4.7) и (4.6), выразим в левой части равенства (6.28) функции $a\left(\mu_{k}\right)$ через $d\left(\mu_{k}\right)$, а в правой части функции $a\left(\lambda_{k}\right)$ через $d\left(\lambda_{k}\right)$. Тогда после несложных преобразований мы получим

$$
\operatorname{det}_{N} \widetilde{G}=\operatorname{det}_{N} \widehat{G}
$$

где

$$
\widehat{G}_{j k}=t\left(\mu_{k}, \lambda_{j}\right) \prod_{a=1}^{N} h\left(\mu_{k}, \lambda_{a}\right)+\varkappa t\left(\lambda_{j}, \mu_{k}\right) \prod_{a=1}^{N} h\left(\lambda_{a}, \mu_{k}\right) \prod_{a=1}^{N} \frac{h\left(\mu_{k}, \mu_{a}\right)}{h\left(\mu_{a}, \mu_{k}\right)},
$$




$$
\widetilde{G}_{j k}=t\left(\mu_{k}, \lambda_{j}\right) \prod_{a=1}^{N} h\left(\mu_{a}, \lambda_{j}\right)+\varkappa t\left(\lambda_{j}, \mu_{k}\right) \prod_{a=1}^{N} h\left(\lambda_{j}, \mu_{a}\right) \prod_{a=1}^{N} \frac{h\left(\lambda_{a}, \lambda_{j}\right)}{h\left(\lambda_{j}, \lambda_{a}\right)} .
$$

ПрЕДЛОЖЕНИЕ 6.3. Соотношение (6.29) является тождеством.

ДокАзАтЕльство [40]. Доказательство этого утверждения похоже на доказательство леммы 6.1 и может быть дано с помощью индукции по $N$. При $N=1$ утверждение очевидно. Предположим, что оно верно для $N-1$. Тогда, рассматривая вычеты детерминантов матриц $\widehat{G}_{j k}$ и $\widetilde{G}_{j k}$ в точках $\lambda_{j}=\mu_{k}$, мы видим, что они сводятся к детерминантам порядка $N-1$, которые равны по индуктивному предположению.

Остается доказать, что детерминанты матриц $\widetilde{G}$ и $\widehat{G}$ не имеют других сингулярностей, кроме полюсов в точках $\lambda_{j}=\mu_{k}$. На первый взгляд можно было бы ожидать, что $\operatorname{det} \widetilde{G}$ имеет простые полюсы в точках $h\left(\lambda_{j}, \lambda_{k}\right)=0, \operatorname{adet} \widehat{G}-$ в точках $h\left(\mu_{j}, \mu_{k}\right)=0$. Легко видеть, однако, что эти сингулярности исчезают. В самом деле, пусть, например, $h\left(\lambda_{1}, \lambda_{2}\right)=0$. Тогда первая строка матрицы $\widetilde{G}$ становится сингулярной:

$$
\widetilde{G}_{1 k} \sim \varkappa t\left(\lambda_{1}, \mu_{k}\right) \prod_{a=1}^{N} h\left(\lambda_{1}, \mu_{a}\right) \prod_{a=1}^{N} \frac{h\left(\lambda_{a}, \lambda_{1}\right)}{h\left(\lambda_{1}, \lambda_{a}\right)} .
$$

Вторая строка матрицы $\widetilde{G}$ приобретает вид

$$
\widetilde{G}_{2 k} \sim t\left(\mu_{k}, \lambda_{2}\right) \prod_{a=1}^{N} h\left(\mu_{a}, \lambda_{2}\right) .
$$

Принимая во внимание, что $t\left(\mu_{k}, \lambda_{2}\right)=t\left(\lambda_{1}, \mu_{k}\right)$ при $h\left(\lambda_{1}, \lambda_{2}\right)=0$, мы видим, что эти две строки пропорциональны друг другу, а следовательно, соответствующий вычет обращается в нуль. Аналогично доказывается, что $\operatorname{det} \widehat{G}$ не имеет полюсов при $h\left(\mu_{j}, \mu_{k}\right)=0$. Предложение 6.3 доказано.

\section{7. Двухкомпонентная модель}

7.1. Определение двухкомпонентной модели. Будем считать, что обобщенная модель, заданная матрицей монодромии $T(\lambda)$ и $R$-матрицей, описывает некоторую одномерную квантовую систему на отрезке $[0, L]$. Как уже отмечалось во введении, матрица монодромии $T(\lambda)$ не является локальным объектом в том смысле, что она не связана с какой-либо конкретной точкой этого интервала. Поэтому включить локальные операторы, описывающие наблюдаемые физической модели, в алгебру (2.2) достаточно сложно. Данное обстоятельство является серьезным препятствием на пути применения алгебраического анзаца Бете к вычислению корреляционных функций локальных операторов.

Тем не менее в ряде случаев указанную трудность удается обойти. Одним из способов сделать это является искусственное деление отрезка произвольным образом на два интервала $[0, x]$ и $[x, L]$. Каждому из этих отрезков мы 
можем сопоставить свои матрицы монодромии $T(\lambda \mid 1)$ и $T(\lambda \mid 2)$. При этом полная матрица монодромии оказывается равной произведению частичных матриц монодромии

$$
T(\lambda)=T(\lambda \mid 2) T(\lambda \mid 1) .
$$

Мы будем называть такую модель обобщенной двухкомпонентной моделью ${ }^{3}$.

Описанный прием позволяет, с одной стороны, включить в рассмотрение операторы, так или иначе зависящие от некоторой внутренней точки $x$, а с другой стороны, по-прежнему работать с матрицами монодромии и $R$-матрицей. По аналогии с формулой (2.1) мы будем обозначать матричные элементы матриц $T(\lambda \mid 1)$ и $T(\lambda \mid 2)$ следующим образом:

$$
T(\lambda \mid j)=\left(\begin{array}{ll}
A_{j}(\lambda) & B_{j}(\lambda) \\
C_{j}(\lambda) & D_{j}(\lambda)
\end{array}\right), \quad j=1,2 .
$$

Каждая из этих матриц удовлетворяет коммутационному соотношению (2.2) с $R$-матрицей (2.8). Операторы, соответствующие различным компонентам, коммутируют друг с другом. Каждая из матриц $T(\lambda \mid j)$ обладает вектором $|0\rangle_{j}$ и дуальным вектором $\left\langle\left. 0\right|_{j}\right.$, причем $\left.\mid 0\right\rangle=|0\rangle_{2} \otimes|0\rangle_{1}$. Формулы для действия операторов на эти векторы аналогичны формулам (2.15), (2.16):

$$
\begin{aligned}
& A_{j}(\lambda)|0\rangle_{j}=a_{j}(\lambda)|0\rangle_{j}, \quad D_{j}(\lambda)|0\rangle=d_{j}(\lambda)|0\rangle_{j}, \quad C_{j}(\lambda)|0\rangle_{j}=0, \\
& \left\langle\left. 0\right|_{j} A_{j}(\lambda)=a_{j}(\lambda)\left\langle\left. 0\right|_{j}, \quad\left\langle\left. 0\right|_{j} D_{j}(\lambda)=d_{j}(\lambda)\left\langle\left. 0\right|_{j}, \quad\left\langle\left. 0\right|_{j} B_{j}(\lambda)=0 .\right.\right.\right.\right.\right.
\end{aligned}
$$

Легко видеть, что $a(\lambda)=a_{1}(\lambda) a_{2}(\lambda)$ и $d(\lambda)=d_{1}(\lambda) d_{2}(\lambda)$.

Покажем теперь, как векторы состояний полной модели выражаются через векторы состояний в каждой из ее компонент [27].

ПРЕДЛОЖЕНИЕ 7.1. Произвольный вектор, соответствующий полному интервалу, выражается через векторы, соответствующие первой и второй компоненте, следующим образом:

$$
\begin{aligned}
& \prod_{j=1}^{N} B\left(\lambda_{j}\right)|0\rangle \\
& =\sum_{\alpha \cup \bar{\alpha}} \prod_{a \in \alpha}\left(a_{2}\left(\lambda_{a}\right) B_{1}\left(\lambda_{a}\right)\right) \prod_{b \in \bar{\alpha}}\left(d_{1}\left(\lambda_{b}\right) B_{2}\left(\lambda_{b}\right)\right)|0\rangle_{2} \otimes|0\rangle_{1} \cdot \prod_{a \in \alpha} \prod_{b \in \bar{\alpha}} f\left(\lambda_{b}, \lambda_{a}\right) .
\end{aligned}
$$

ДокАЗАТЕЛьСтво. Формула (7.3) может быть доказана индукцией по $N$. Для $N=1$ соотношение (7.3) справедливо. Пусть оно верно для $N-1$. Тогда

$$
\begin{aligned}
& \prod_{j=1}^{N} B\left(\lambda_{j}\right)|0\rangle=\left(A_{2}\left(\lambda_{N}\right) B_{1}\left(\lambda_{N}\right)+B_{2}\left(\lambda_{N}\right) D_{1}\left(\lambda_{N}\right)\right) \\
& \times \sum_{\alpha \cup \bar{\alpha}} \prod_{a \in \alpha}\left(a_{2}\left(\lambda_{a}\right) B_{1}\left(\lambda_{a}\right)\right) \prod_{b \in \bar{\alpha}}\left(d_{1}\left(\lambda_{b}\right) B_{2}\left(\lambda_{b}\right)\right)|0\rangle_{2} \otimes|0\rangle_{1} \cdot \prod_{a \in \alpha} \prod_{b \in \bar{\alpha}} f\left(\lambda_{b}, \lambda_{a}\right),
\end{aligned}
$$

\footnotetext{
${ }^{3}$ В работе [27], где эта модель была введена впервые, она называлась обобщенной двухузельной моделью. На наш взгляд такой термин может вызвать недоразумения, в особенности при его применении к решеточным моделям.
} 
где сумма берется по разбиениям набора из $N-1$ переменных $\lambda_{1}, \ldots, \lambda_{N-1}$. Подействуем операторами $A_{2}\left(\lambda_{N}\right)$ и $D_{1}\left(\lambda_{N}\right)$ на получившиеся состояния и рассмотрим сначала вклады, возникающие в случае, когда операторы $A_{2}$ и $D_{1}$ обмениваются своими аргументами с операторами $B_{2}$ и $B_{1}$. Пользуясь формулами (3.12), (3.16), находим вклад от оператора $A_{2}$ :

$$
\begin{aligned}
& \sum_{\alpha \cup \bar{\alpha}} \sum_{k \in \bar{\alpha}} g\left(\lambda_{N}, \lambda_{k}\right) a_{2}\left(\lambda_{k}\right) d_{1}\left(\lambda_{k}\right) B_{1}\left(\lambda_{N}\right) B_{2}\left(\lambda_{N}\right) \\
& \times \prod_{a \in \alpha}\left(a_{2}\left(\lambda_{a}\right) B_{1}\left(\lambda_{a}\right)\right) \prod_{\substack{b \in \bar{\alpha} \\
b \neq k}}\left(d_{1}\left(\lambda_{b}\right) B_{2}\left(\lambda_{b}\right)\right)|0\rangle_{2} \otimes|0\rangle_{1} \cdot \prod_{\substack{b \in \bar{\alpha} \\
b \neq k}} f\left(\lambda_{b}, \lambda_{k}\right) \prod_{a \in \alpha} \prod_{b \in \bar{\alpha}} f\left(\lambda_{b}, \lambda_{a}\right) .
\end{aligned}
$$

Аналогично, используя (3.17), (3.18), находим вклад от оператора $D_{1}$ :

$$
\begin{aligned}
& \sum_{\alpha \cup \bar{\alpha}} \sum_{k \in \alpha} g\left(\lambda_{k}, \lambda_{N}\right) a_{2}\left(\lambda_{k}\right) d_{1}\left(\lambda_{k}\right) B_{1}\left(\lambda_{N}\right) B_{2}\left(\lambda_{N}\right) \\
& \times \prod_{\substack{a \in \alpha \\
b \neq k}}\left(a_{2}\left(\lambda_{a}\right) B_{1}\left(\lambda_{a}\right)\right) \prod_{b \in \bar{\alpha}}\left(d_{1}\left(\lambda_{b}\right) B_{2}\left(\lambda_{b}\right)\right)|0\rangle_{2} \otimes|0\rangle_{1} \cdot \prod_{\substack{b \in \alpha \\
b \neq k}} f\left(\lambda_{k}, \lambda_{b}\right) \prod_{a \in \alpha} \prod_{b \in \bar{\alpha}} f\left(\lambda_{b}, \lambda_{a}\right) .
\end{aligned}
$$

Вводя в формуле (7.6) новые разбиения $\{\lambda\}_{\alpha^{\prime}}=\{\lambda\}_{\alpha} \backslash \lambda_{k}$ и $\{\lambda\}_{\bar{\alpha}^{\prime}}=\{\lambda\}_{\bar{\alpha}} \cup \lambda_{k}$, мы видим, что слагаемые (7.6) и (7.5) в точности сокращают друг друга. Таким образом, операторы $A_{2}$ и $D_{1}$ действуют на получившиеся состояния только по первой схеме коммутации, т. е. сохраняя свои аргументы. Учитывая (3.12), (3.13), (3.17) и (3.18), мы немедленно получаем доказательство утверждения. Предложение 7.1 доказано.

Формула, аналогичная (7.3), существует и для дуальных состояний

$$
\begin{aligned}
& \langle 0| \prod_{j=1}^{N} C\left(\lambda_{j}\right) \\
& =\left\langle0 | _ { 1 } \otimes \left\langle\left. 0\right|_{2} \sum_{\alpha \cup \bar{\alpha}} \prod_{a \in \alpha}\left(a_{1}\left(\lambda_{a}\right) C_{2}\left(\lambda_{a}\right)\right) \prod_{b \in \bar{\alpha}}\left(d_{2}\left(\lambda_{b}\right) C_{1}\left(\lambda_{b}\right)\right) \cdot \prod_{a \in \alpha} \prod_{b \in \bar{\alpha}} f\left(\lambda_{b}, \lambda_{a}\right) .\right.\right.
\end{aligned}
$$

7.2. Матричный элемент оператора числа частиц на отрезке $[0, x]$. Перейдем теперь к задаче о вычислении матричных элементов локальных операторов. В качестве примера мы рассмотрим оператор числа частиц $Q_{1}$ на отрезке $[0, x]$, введенный в работе [27]. Этот оператор можно задать с помощью его коммутационных соотношений с элементами матрицы $T(\lambda \mid 1)$ :

$$
\left[Q_{1}, B_{1}(\lambda)\right]=B_{1}(\lambda), \quad\left[Q_{1}, C_{1}(\lambda)\right]=-C_{1}(\lambda)
$$

Остальные коммутационные соотношения тривиальны. Действие оператора $Q_{1}$ на псевдовакуум дается формулами

$$
Q_{1}|0\rangle_{1}=0, \quad\left\langle\left. 0\right|_{1} Q_{1}=0 .\right.
$$


По причинам технического характера удобнее иметь дело с оператором $e^{\beta Q_{1}}$, где $\beta$ - некоторое комплексное число, и рассматривать производящую функцию

$$
\left\langle e^{\beta Q_{1}}\right\rangle=\frac{\left\langle\psi(\{\lambda\})\left|e^{\beta Q_{1}}\right| \psi(\{\lambda\})\right\rangle}{\langle\psi(\{\lambda\}) \mid \psi(\{\lambda\})\rangle}
$$

Здесь $|\psi(\{\lambda\})\rangle$ - одно из собственных состояний гамильтониана, например основное состояние. Производящая функция (7.10) является одним из самых простых объектов, которые могут быть вычислены в рамках общей схемы алгебраического анзаца Бете. Вместе с тем данный пример оказывается вполне содержательным с физической точки зрения. Ниже, на примере $X X Z$-цепочки Гейзенберга, мы рассмотрим корреляционные функции, которые могут быть вычислены, исходя из среднего (7.10).

Пока же мы продолжим изучение оператора $Q_{1}$ в рамках обобщенной двухкомпонентной модели. Рассмотрим следующий матричный элемент:

$$
\left\langle\left\langle Q_{1}^{\beta}\right\rangle\right\rangle=\left\langle 0\left|\prod_{j=1}^{N} \frac{C\left(\mu_{j}\right)}{d\left(\mu_{j}\right)} \cdot e^{\beta Q_{1}} \cdot \prod_{j=1}^{N} \frac{B\left(\lambda_{j}\right)}{d\left(\lambda_{j}\right)}\right| 0\right\rangle \cdot \prod_{j>k}^{N} g^{-1}\left(\lambda_{j}, \lambda_{k}\right) g^{-1}\left(\mu_{k}, \mu_{j}\right),
$$

где $\{\lambda\}$ и $\{\mu\}-$ произвольные комплексные числа. Тогда в силу (7.3), (7.7) и (7.8) имеем

$$
\begin{aligned}
\left\langle\left\langle Q_{1}^{\beta}\right\rangle\right\rangle & =\sum_{\substack{\alpha \cup \bar{\alpha} \\
\gamma \cup \bar{\gamma}}} e^{\beta n}\left\langle\left. 0\right|_{1} \prod_{j \in \gamma}^{N} \frac{C_{1}\left(\mu_{j}\right)}{d_{1}\left(\mu_{j}\right)} \prod_{j \in \alpha}^{N} \frac{B_{1}\left(\lambda_{j}\right)}{d_{1}\left(\lambda_{j}\right)} \mid 0\right\rangle_{1}\left\langle\left. 0\right|_{2} \prod_{j \in \bar{\gamma}}^{N} \frac{C_{2}\left(\mu_{j}\right)}{d_{2}\left(\mu_{j}\right)} \prod_{j \in \bar{\alpha}}^{N} \frac{B_{2}\left(\lambda_{j}\right)}{d_{2}\left(\lambda_{j}\right)} \mid 0\right\rangle_{2} \\
& \times \prod_{\substack{a>b \\
a, b \in \alpha}} g^{-1}\left(\lambda_{a}, \lambda_{b}\right) \prod_{\substack{a<b \\
a, b \in \gamma}} g^{-1}\left(\mu_{a}, \mu_{b}\right) \prod_{\substack{a>b \\
a, b \in \bar{\alpha}}} g^{-1}\left(\lambda_{a}, \lambda_{b}\right) \prod_{\substack{a<b \\
a, b \in \gamma}} g^{-1}\left(\mu_{a}, \mu_{b}\right) \\
& \times(-1)^{P_{\alpha}+P_{\gamma}} \prod_{a \in \alpha} m\left(\lambda_{a}\right) \prod_{a \in \bar{\gamma}} l\left(\mu_{a}\right) \prod_{a \in \alpha} \prod_{b \in \bar{\alpha}} h\left(\lambda_{b}, \lambda_{a}\right) \prod_{a \in \gamma} \prod_{b \in \bar{\gamma}} h\left(\mu_{a}, \mu_{b}\right) .
\end{aligned}
$$

Здесь $n$ означает количество элементов в наборе $\{\alpha\}$ (или $\{\gamma\}$ ), и мы также ввели новые функции

$$
l(\lambda)=\frac{a_{1}(\lambda)}{d_{1}(\lambda)}, \quad m(\lambda)=\frac{a_{2}(\lambda)}{d_{2}(\lambda)}, \quad r(\lambda)=\frac{a(\lambda)}{d(\lambda)}=l(\lambda) m(\lambda) .
$$

Мы видим, что вычисление матричного элемента оператора $Q_{1}$ сводится к вычислению скалярных произведений в первой и второй компоненте. Для каждого из этих скалярных произведений мы можем воспользоваться формулой (5.25) с соответствующей заменой функций $r(\lambda)$ на $l(\lambda)$ или $m(\lambda)$. В результате мы получаем сумму по четырем разбиениям набора $\{\lambda\}$ и четырем 

разбиениям набора $\{\mu\}$ :

$$
\begin{aligned}
& \left\langle\left\langle Q_{1}^{\beta}\right\rangle\right\rangle=\sum_{\substack{\alpha+\cup \alpha_{-} \cup \bar{\alpha}_{+} \cup \bar{\alpha}_{-} \\
\gamma_{+} \cup \gamma_{-} \cup \bar{\gamma}_{+} \cup \bar{\gamma}_{-}}} \prod_{a \in \gamma_{+} \cup \bar{\gamma}_{-}} l\left(\mu_{a}\right) \prod_{a \in \alpha_{+} \cup \bar{\alpha}_{-}} m\left(\lambda_{a}\right) \prod_{a \in \bar{\gamma}_{+}} r\left(\mu_{a}\right) \prod_{a \in \alpha_{-}} r\left(\lambda_{a}\right) \\
& \times e^{\beta\left(n_{+}+n_{-}\right)} \operatorname{det}_{\substack{j \in \alpha_{+} \\
k \in \gamma_{+}}} t\left(\lambda_{j}, \mu_{k}\right) \operatorname{det}_{\substack{j \in \alpha_{-} \\
k \in \gamma_{-}}} t\left(\mu_{k}, \lambda_{j}\right) \operatorname{det}_{\substack{j \in \bar{\alpha}_{+} \\
k \in \bar{\gamma}_{+}}} t\left(\lambda_{j}, \mu_{k}\right) \operatorname{det}_{\substack{j \in \bar{\alpha}_{-} \\
k \in \bar{\gamma}_{-}}} t\left(\mu_{k}, \lambda_{j}\right) \\
& \times \prod_{a \in \gamma_{+}} \prod_{b \in \alpha_{+}} h\left(\lambda_{b}, \mu_{a}\right) \prod_{a \in \alpha_{-}} \prod_{b \in \gamma_{-}} h\left(\mu_{b}, \lambda_{a}\right) \prod_{a \in \alpha_{-}} \prod_{b \in \alpha_{+}} h\left(\lambda_{b}, \lambda_{a}\right) \prod_{a \in \gamma_{+}} \prod_{b \in \gamma_{-}} h\left(\mu_{b}, \mu_{a}\right) \\
& \times \prod_{a \in \bar{\gamma}_{+}} \prod_{b \in \bar{\alpha}_{+}} h\left(\lambda_{b}, \mu_{a}\right) \prod_{a \in \bar{\alpha}_{-}} \prod_{b \in \bar{\gamma}_{-}} h\left(\mu_{b}, \lambda_{a}\right) \prod_{a \in \bar{\alpha}_{-}} \prod_{b \in \bar{\alpha}_{+}} h\left(\lambda_{b}, \lambda_{a}\right) \prod_{a \in \bar{\gamma}_{+}} \prod_{b \in \bar{\gamma}_{-}} h\left(\mu_{b}, \mu_{a}\right) \\
& \times(-1)^{P_{\alpha}+P_{\gamma}} \prod_{a \in \alpha_{+} \cup \alpha_{-}} \prod_{b \in \bar{\alpha}_{+} \cup \bar{\alpha}_{-}} h\left(\lambda_{b}, \lambda_{a}\right) \prod_{a \in \gamma_{+} \cup \gamma_{-}} \prod_{b \in \bar{\gamma}_{+} \cup \bar{\gamma}_{-}} h\left(\mu_{a}, \mu_{b}\right) .
\end{aligned}
$$

Здесь, как обычно, $P_{\alpha}$ и $P_{\gamma}$ означают четность перестановок

$$
\begin{gathered}
P\left(\left\{\alpha_{+}\right\},\left\{\alpha_{-}\right\},\left\{\bar{\alpha}_{+}\right\},\left\{\bar{\alpha}_{-}\right\}\right)=1, \ldots, N, \\
P\left(\left\{\gamma_{+}\right\},\left\{\gamma_{-}\right\},\left\{\bar{\gamma}_{+}\right\},\left\{\bar{\gamma}_{-}\right\}\right)=1, \ldots, N .
\end{gathered}
$$

Количество элементов в наборах $\left\{\alpha_{+}\right\}$и $\left\{\alpha_{-}\right\}$обозначено соответственно через $n_{+}$и $n_{-}$.

7.3. Частный случай матричного элемента оператора $Q_{1}$. Выражение (7.14) для матричного элемента оператора $Q_{1}$ крайне громоздко и само по себе непригодно для практических вычислений. Однако, как и в случае скалярного произведения, формула для матричного элемента резко упрощается, если одно из состояний является собственной функцией трансферматрицы. Имеет место следующее утверждение [40].

ПрЕДЛОЖЕНИЕ 7.2. Пусть параметры $\{\mu\}$ суть произвольные комплекснъе числа, а набор параметров $\{\lambda\}$ удовлетворяет системе уравнений Беme (4.6). Тогда

$$
\begin{aligned}
\left\langle\left\langle Q_{1}^{\beta}\right\rangle\right\rangle=\frac{1}{N !} \oint_{\Gamma(\{\mu\} \cup\{\lambda\})} \prod_{j=1}^{N} \frac{d z_{j}}{2 \pi i} \prod_{j=1}^{N} \frac{l\left(z_{j}\right) l^{-1}\left(\lambda_{j}\right) d\left(z_{j}\right)}{d\left(\lambda_{j}\right) d\left(\mu_{j}\right)} \\
\times \frac{\operatorname{det}_{N} \Omega_{\varkappa}\left(z_{k}, \mu_{j}\right) \operatorname{det}_{N} \Omega_{\varkappa}\left(z_{k}, \lambda_{j}\right)}{\prod_{j=1}^{N} \mathscr{Y}_{\varkappa}\left(z_{j} \mid\{z\}\right)},
\end{aligned}
$$

где $\varkappa=e^{\beta}$, а контуры интегрирования $\Gamma(\{\mu\} \cup\{\lambda\})$ охватывают полюсы функuий $\operatorname{det} \Omega_{\varkappa}$ в точках $\{\lambda\} u\{\mu\}$ и не содержат никаких других особенностей подынтегрального выражения.

ДокАЗАТЕЛЬство. Как и в случае скалярного произведения, выразим в (7.14) функции $a\left(\lambda_{a}\right)$ через $d\left(\lambda_{a}\right)$ с помощью уравнений (4.6). Вводя обозначения

$$
\begin{array}{ll}
\alpha_{0}=\alpha_{+} \cup \bar{\alpha}_{-}, & \bar{\alpha}_{0}=\bar{\alpha}_{+} \cup \alpha_{-}, \\
\gamma_{0}=\gamma_{+} \cup \bar{\gamma}_{-}, & \bar{\gamma}_{0}=\bar{\gamma}_{+} \cup \gamma_{-},
\end{array}
$$


мы получаем

$$
\begin{aligned}
& \left\langle\left\langle Q_{1}^{\beta}\right\rangle\right\rangle=\sum_{\substack{\alpha+\cup \alpha_{-} \cup \bar{\alpha}_{+} \cup \bar{\alpha}_{-} \\
\gamma_{+} \cup \gamma_{-} \cup \bar{\gamma}_{+} \cup \bar{\gamma}_{-}}}(-1)^{P_{\alpha}+P_{\gamma}} \prod_{a \in \gamma_{0}} l\left(\mu_{a}\right) \prod_{a \in \alpha_{0}} m\left(\lambda_{a}\right) \cdot e^{\beta n_{+}} \operatorname{det}_{\substack{j \in \alpha_{+} \\
k \in \gamma_{+}}} t\left(\lambda_{j}, \mu_{k}\right) \\
& \times \operatorname{det}_{\substack{j \in \bar{\alpha}_{-} \\
k \in \bar{\gamma}_{-}}} t\left(\mu_{k}, \lambda_{j}\right) \prod_{a \in \bar{\alpha}_{0}} \prod_{b \in \alpha_{0}} h\left(\lambda_{a}, \lambda_{b}\right) \prod_{b \in \gamma_{-}} \prod_{a \in \gamma_{0}} h\left(\mu_{b}, \mu_{a}\right) \prod_{b \in \bar{\gamma}_{+}} \prod_{a \in \gamma_{0}} h\left(\mu_{a}, \mu_{b}\right) \\
& \times \prod_{a \in \gamma_{+}} \prod_{b \in \alpha_{+}} h\left(\lambda_{b}, \mu_{a}\right) \prod_{a \in \bar{\alpha}_{-}} \prod_{b \in \bar{\gamma}_{-}} h\left(\mu_{b}, \lambda_{a}\right) \prod_{a \in \bar{\alpha}_{-}} \prod_{b \in \alpha_{+}} h\left(\lambda_{a}, \lambda_{b}\right) \prod_{a \in \gamma_{+}} \prod_{b \in \bar{\gamma}_{-}} h\left(\mu_{a}, \mu_{b}\right) \\
& \times e^{\beta n_{-}}(-1)^{(N-1) n_{-}} \prod_{a \in \bar{\gamma}_{+}} r\left(\mu_{a}\right) \operatorname{det}_{\substack{j \in \alpha_{-} \\
k \in \gamma_{-}}} t\left(\mu_{k}, \lambda_{j}\right) \operatorname{det}_{\substack{j \in \bar{\alpha}_{+} \\
k \in \bar{\gamma}_{+}}} t\left(\lambda_{j}, \mu_{k}\right) \\
& \times \prod_{a \in \alpha_{-}} \prod_{b \in \gamma_{-}} h\left(\mu_{b}, \lambda_{a}\right) \prod_{a \in \bar{\gamma}_{+}} \prod_{b \in \bar{\alpha}_{+}} h\left(\lambda_{b}, \mu_{a}\right) \prod_{a \in \alpha_{-}} \prod_{b \in \bar{\alpha}_{+}} h\left(\lambda_{a}, \lambda_{b}\right) \prod_{a \in \gamma-} \prod_{b \in \bar{\gamma}_{+}} h\left(\mu_{a}, \mu_{b}\right) .
\end{aligned}
$$

Заметим, что подмножества $\{\lambda\}_{\alpha_{-}}$и $\{\lambda\}_{\bar{\alpha}_{+}}$входят по отдельности только в две последние строки формулы (7.18). Сумму по этим разбиениям (при остальных фиксированных) можно преобразовать с помощью леммы 6.1:

$$
\begin{aligned}
& \sum_{\alpha-\cup \bar{\alpha}_{+}}(-1)^{P_{\alpha}} \operatorname{det}_{\substack{j \in \alpha_{-} \\
k \in \gamma_{-}}} t\left(\mu_{k}, \lambda_{j}\right) \operatorname{det}_{\substack{j \in \bar{\alpha}_{+} \\
k \in \bar{\gamma}_{+}}} t\left(\lambda_{j}, \mu_{k}\right) \\
& \quad \times \prod_{a \in \alpha_{-}} \prod_{b \in \gamma_{-}} h\left(\mu_{b}, \lambda_{a}\right) \prod_{a \in \bar{\gamma}_{+}} \prod_{b \in \bar{\alpha}_{+}} h\left(\lambda_{b}, \mu_{a}\right) \prod_{a \in \alpha_{-}} \prod_{b \in \bar{\alpha}_{+}} h\left(\lambda_{a}, \lambda_{b}\right) \prod_{a \in \gamma_{-}} \prod_{b \in \bar{\gamma}_{+}} h\left(\mu_{a}, \mu_{b}\right) \\
& =\sum_{\alpha-\cup \bar{\alpha}_{+}}(-1)^{P_{\alpha}} \operatorname{det}_{\substack{j \in \alpha_{-} \\
k \in \gamma_{-}}} t\left(\mu_{k}, \lambda_{j}\right) \operatorname{det}_{\substack{\operatorname{det}_{j \in \bar{\alpha}_{+}} \\
k \in \bar{\gamma}_{+}}} t\left(\lambda_{j}, \mu_{k}\right) \\
& \quad \times \prod_{b \in \bar{\gamma}_{+}} \prod_{a \in \bar{\alpha}_{0}} h\left(\lambda_{a}, \mu_{b}\right) \prod_{b \in \gamma_{-}} \prod_{a \in \bar{\alpha}_{0}} h\left(\mu_{b}, \lambda_{a}\right) .
\end{aligned}
$$

Подставляя это выражение в формулу (7.18), мы видим, что сумма по разбиениям $\left\{\alpha_{-}\right\},\left\{\bar{\alpha}_{+}\right\},\left\{\gamma_{-}\right\},\left\{\bar{\gamma}_{+}\right\}$в точности дает формулу Лапласа (6.1). Таким образом, мы получаем

$$
\begin{aligned}
& \left\langle\left\langle Q_{1}^{\beta}\right\rangle\right\rangle=\sum_{\substack{\alpha+\cup \bar{\alpha}_{-} \cup \bar{\alpha}_{0} \\
\gamma_{+} \cup \bar{\gamma}_{-} \cup \bar{\gamma}_{0}}} \prod_{a \in \gamma_{0}} l\left(\mu_{a}\right) \prod_{a \in \alpha_{0}} m\left(\lambda_{a}\right) \cdot e^{\beta n_{+}} \cdot \operatorname{det}_{\substack{j \in \alpha_{+} \\
k \in \gamma_{+}}} t\left(\lambda_{j}, \mu_{k}\right) \operatorname{det}_{\substack{j \in \bar{\alpha}_{-} \\
k \in \bar{\gamma}_{-}}} t\left(\mu_{k}, \lambda_{j}\right) \\
& \times(-1)^{P_{\alpha}+P_{\gamma}} \operatorname{det}_{\substack{j \in \bar{\alpha}_{0} \\
k \in \bar{\gamma}_{0}}}\left[r\left(\mu_{k}\right) t\left(\lambda_{j}, \mu_{k}\right) \prod_{a \in \bar{\alpha}_{0}} h\left(\lambda_{a}, \mu_{k}\right) \prod_{a \in \gamma_{0}} h\left(\mu_{a}, \mu_{k}\right)\right. \\
& \left.-e^{\beta}(-1)^{N} t\left(\mu_{k}, \lambda_{j}\right) \prod_{a \in \bar{\alpha}_{0}} h\left(\mu_{k}, \lambda_{a}\right) \prod_{a \in \gamma_{0}} h\left(\mu_{k}, \mu_{a}\right)\right] \cdot \prod_{a \in \bar{\alpha}_{0}} \prod_{b \in \alpha_{0}} h\left(\lambda_{a}, \lambda_{b}\right) \\
& \quad \times \prod_{a \in \gamma_{+}} \prod_{b \in \alpha_{+}} h\left(\lambda_{b}, \mu_{a}\right) \prod_{a \in \bar{\alpha}_{-}} \prod_{b \in \bar{\gamma}_{-}} h\left(\mu_{b}, \lambda_{a}\right) \prod_{a \in \bar{\alpha}_{-}} \prod_{b \in \alpha_{+}} h\left(\lambda_{a}, \lambda_{b}\right) \prod_{a \in \gamma_{+}} \prod_{b \in \bar{\gamma}_{-}} h\left(\mu_{a}, \mu_{b}\right) .
\end{aligned}
$$


Теперь при фиксированных разбиениях $\bar{\alpha}_{0}$ и $\bar{\gamma}_{0}$ мы можем просуммировать по оставшимся разбиениям. Снова используя лемму 6.1, мы находим

$$
\begin{aligned}
& \sum_{\substack{\alpha+\cup \bar{\alpha}_{-} \\
\gamma+\cup \bar{\gamma}_{-}}}(-1)^{P_{\alpha}+P_{\gamma}} e^{\beta n_{+}} \cdot \operatorname{det}_{\substack{j \in \alpha_{+} \\
k \in \gamma_{+}}} t\left(\lambda_{j}, \mu_{k}\right) \operatorname{det}_{\substack{j \in \bar{\alpha}_{-} \\
k \in \bar{\gamma}_{-}}} t\left(\mu_{k}, \lambda_{j}\right) \cdot \prod_{a \in \gamma_{+}} \prod_{b \in \gamma_{0}} \frac{h\left(\mu_{a}, \mu_{b}\right)}{h\left(\mu_{b}, \mu_{a}\right)} \\
& \times \prod_{a \in \gamma_{+}} \prod_{b \in \alpha_{+}} h\left(\lambda_{b}, \mu_{a}\right) \prod_{a \in \bar{\alpha}_{-}} \prod_{b \in \bar{\gamma}_{-}} h\left(\mu_{b}, \lambda_{a}\right) \prod_{a \in \bar{\alpha}_{-}} \prod_{b \in \alpha_{+}} h\left(\lambda_{a}, \lambda_{b}\right) \prod_{a \in \gamma_{+}} \prod_{b \in \bar{\gamma}_{-}} h\left(\mu_{b}, \mu_{a}\right) \\
& =\sum_{\substack{\alpha+\cup \bar{\alpha}_{-} \\
\gamma+\cup \bar{\gamma}_{-}}}(-1)^{P_{\alpha}+P_{\gamma}} e^{\beta n_{+}} \cdot \operatorname{det}_{\substack{j \in \alpha_{+} \\
k \in \gamma_{+}}} t\left(\lambda_{j}, \mu_{k}\right) \operatorname{det}_{\substack{j \in \bar{\alpha}_{-} \\
k \in \bar{\gamma}_{-}}} t\left(\mu_{k}, \lambda_{j}\right) \cdot \prod_{a \in \gamma_{+}} \prod_{b \in \gamma_{0}} \frac{h\left(\mu_{a}, \mu_{b}\right)}{h\left(\mu_{b}, \mu_{a}\right)} \\
& \times \prod_{b \in \gamma_{+}} \prod_{a \in \alpha_{0}} h\left(\lambda_{a}, \mu_{b}\right) \prod_{b \in \bar{\gamma}_{-}} \prod_{a \in \alpha_{0}} h\left(\mu_{b}, \lambda_{a}\right) \\
& =\operatorname{det}_{\substack{j \in \alpha_{0} \\
k \in \gamma_{0}}}\left[t\left(\mu_{k}, \lambda_{j}\right) \prod_{a \in \alpha_{0}} h\left(\mu_{k}, \lambda_{a}\right)+e^{\beta} t\left(\lambda_{j}, \mu_{k}\right) \prod_{a \in \alpha_{0}} h\left(\lambda_{a}, \mu_{k}\right) \prod_{a \in \gamma_{0}} \frac{h\left(\mu_{k}, \mu_{a}\right)}{h\left(\mu_{a}, \mu_{k}\right)}\right] .
\end{aligned}
$$

Применяя теперь тождество (6.29) из предложения 6.3, мы приходим к следующему выражению:

$$
\begin{aligned}
& \left\langle\left\langle Q_{1}^{\beta}\right\rangle\right\rangle=\sum_{\substack{\alpha_{0} \cup \bar{\alpha}_{0} \\
\gamma_{0} \cup \bar{\gamma}_{0}}}(-1)^{P_{\alpha}+P_{\gamma}} \prod_{a \in \gamma_{0}} l\left(\mu_{a}\right) \prod_{a \in \alpha_{0}} m\left(\lambda_{a}\right) \cdot \prod_{a \in \bar{\alpha}_{0}} \prod_{b \in \alpha_{0}} h\left(\lambda_{a}, \lambda_{b}\right) \\
& \times \operatorname{det}_{\substack{j \in \alpha_{0} \\
k \in \gamma_{0}}}\left[t\left(\mu_{k}, \lambda_{j}\right) \prod_{a \in \gamma_{0}} h\left(\mu_{a}, \lambda_{j}\right)+e^{\beta} t\left(\lambda_{j}, \mu_{k}\right) \prod_{a \in \gamma_{0}} h\left(\lambda_{j}, \mu_{a}\right) \prod_{a \in \alpha_{0}} \frac{h\left(\lambda_{a}, \lambda_{j}\right)}{h\left(\lambda_{j}, \lambda_{a}\right)}\right] \\
& \quad \times \operatorname{det}_{\substack{j \in \bar{\alpha}_{0} \\
k \in \bar{\gamma}_{0}}}\left[r\left(\mu_{k}\right) t\left(\lambda_{j}, \mu_{k}\right) \prod_{a \in \bar{\alpha}_{0}} h\left(\lambda_{a}, \mu_{k}\right) \prod_{a \in \gamma_{0}} h\left(\mu_{a}, \mu_{k}\right)\right. \\
& \left.-e^{\beta}(-1)^{N} t\left(\mu_{k}, \lambda_{j}\right) \prod_{a \in \bar{\alpha}_{0}} h\left(\mu_{k}, \lambda_{a}\right) \prod_{a \in \gamma_{0}} h\left(\mu_{k}, \mu_{a}\right)\right] .
\end{aligned}
$$

С другой стороны, легко видеть, что, вычисляя интеграл (7.16) по вычетам в точках $z_{k}=\lambda_{j}$ и $z_{k}=\mu_{j}$, мы в точности воспроизводим формулу (7.22). Предложение 7.2 доказано.

\section{8. $X X Z$-цепочка Гейзенберга спина $1 / 2$}

В качестве примера применения разработанной выше техники мы рассмотрим вычисление корреляционных функций в $X X Z$-цепочке Гейзенберга [41]. Модель представляет собой замкнутую одномерную цепочку частиц спина 1/2, в которой взаимодействуют ближайшие соседи. Гамильтониан взаимодействия имеет вид

$$
H=\sum_{m=1}^{M}\left(\sigma_{m}^{x} \sigma_{m+1}^{x}+\sigma_{m}^{y} \sigma_{m+1}^{y}+\Delta\left(\sigma_{m}^{z} \sigma_{m+1}^{z}-1\right)\right) .
$$


Здесь $\Delta$ - вещественное число, называемое параметром анизотропии, $\sigma_{m}^{x, y, z}$ операторы спина $1 / 2$, описывающие частицу в узле с номером $m$. Общее число узлов цепочки $M$ предполагается четным. Пространство состояний данного гамильтониана имеет вид $\mathscr{H}=\bigotimes_{m=1}^{M} \mathscr{H}_{m}$, где $\mathscr{H}_{m} \sim \mathbb{C}^{2}$ носит название локального квантового пространства в узле $m$. Операторы $\sigma_{m}^{x, y, z}$ действуют как соответствующие матрицы Паули в пространстве $\mathscr{H}_{m}$ и как единичный оператор во всех остальных пространствах.

$R$-матрица данной модели дается формулой (2.8), причем $\Delta=\operatorname{ch} \eta$. Для построения матрицы монодромии мы введем сначала так называемый $L$-оператор в узле $m$, отождествляя одно из векторных пространств $R$-матрицы с локальным квантовым пространством $\mathscr{H}_{m}$,

$$
L_{m}(\lambda)=R_{0 m}\left(\lambda-\frac{\eta}{2}\right) .
$$

Здесь $R_{0 m}$ действует в $\mathbb{C}^{2} \otimes \mathscr{H}_{m}$. Матрица монодромии $T(\lambda)$ строится как упорядоченное произведение $L$-операторов по всем узлам цепочки [42]:

$$
T(\lambda)=\left(\begin{array}{ll}
A(\lambda) & B(\lambda) \\
C(\lambda) & D(\lambda)
\end{array}\right)=L_{M}(\lambda) \ldots L_{2}(\lambda) L_{1}(\lambda) .
$$

Гамильтониан (8.1) связан с трансферматрицей $\mathscr{T}(\lambda)$ следующим образом:

$$
H=\left.2 \operatorname{sh} \eta \frac{d \mathscr{T}(\lambda)}{d \lambda} \mathscr{T}^{-1}(\lambda)\right|_{\lambda=\eta / 2}+\text { const. }
$$

Ниже мы будем рассматривать неоднородную $X X Z$-цепочку [43], в которой

$$
\begin{aligned}
L_{m}(\lambda) & =L_{m}\left(\lambda, \xi_{m}\right)=R_{0 m}\left(\lambda-\xi_{m}\right), \\
T(\lambda) & =L_{M}\left(\lambda, \xi_{M}\right) \ldots L_{2}\left(\lambda, \xi_{2}\right) L_{1}\left(\lambda, \xi_{1}\right),
\end{aligned}
$$

где $\xi_{m}$ суть некоторые комплексные числа, называемые параметрами неоднородности. В случае общего положения параметров $\xi_{m}$ такая модель не имеет физического содержания (в частности, оператор, построенный по формуле (8.4), не является эрмитовым и локальным), однако гораздо более удобна с технической точки зрения. В однородном пределе $\xi_{m}=\eta / 2$ мы возвращаемся к исходной $X X Z$-цепочке.

Вектором псевдовакуума $|0\rangle$ является состояние со всеми спинами вверх:

$$
|0\rangle=\bigotimes_{k=1}^{M}\left(\begin{array}{l}
1 \\
0
\end{array}\right)_{k}, \quad\langle 0|=| 0\rangle^{T} .
$$

Вакуумные собственные значения матрицы монодромии $a(\lambda)$ и $d(\lambda)$ имеют вид

$$
\begin{aligned}
& a(\lambda)=\prod_{a=1}^{M} \frac{\operatorname{sh}\left(\lambda-\xi_{a}+\eta\right)}{\operatorname{sh}\left(\lambda-\xi_{a}\right)} \\
& d(\lambda)=1 .
\end{aligned}
$$


Оператор $B$, действуя на состояние с общим спином $S$, переводит его в состояние со спином $S-1$; в частности, действуя на псевдовакуум, оператор $B$ переводит его в линейную комбинацию состояний, в каждом из которых ровно одна частица имеет спин вниз. Для того чтобы сохранить за оператором $B$ смысл оператора рождения, состояние с $N$ спинами вниз называют состоянием с $N$ квазичастицами. Тогда оператор числа спинов вниз в полной цепочке

$$
Q=\frac{1}{2} \sum_{k=1}^{M}\left(1-\sigma_{k}^{z}\right)
$$

можно интерпретировать как оператор числа квазичастиц, и он имеет следующие ненулевые коммутационные соотношения с элементами матрицы монодромии:

$$
[Q, B(\lambda)]=B(\lambda), \quad[Q, C(\lambda)]=-C(\lambda) .
$$

Выбирая произвольным образом узел с номером $m$ и полагая

$$
T(\lambda \mid 1)=L_{m}(\lambda) \cdots L_{1}(\lambda), \quad T(\lambda \mid 2)=L_{M}(\lambda) \cdots L_{m+1}(\lambda)
$$

мы получаем двухкомпонентную модель [44]. Очевидно, что оператор числа квазичастиц в первой компоненте имеет вид

$$
Q_{1}=\frac{1}{2} \sum_{k=1}^{m}\left(1-\sigma_{k}^{z}\right)
$$

a

$$
l(\lambda)=\prod_{a=1}^{m} \frac{\operatorname{sh}\left(\lambda-\xi_{a}+\eta\right)}{\operatorname{sh}\left(\lambda-\xi_{a}\right)}, \quad m(\lambda)=\prod_{a=m+1}^{M} \frac{\operatorname{sh}\left(\lambda-\xi_{a}+\eta\right)}{\operatorname{sh}\left(\lambda-\xi_{a}\right)} .
$$

Зная производящую функцию (7.10) $\left\langle e^{\beta Q_{1}}\right\rangle$, можно вычислять различные корреляционные функции третьих компонент спинов, например,

$$
\begin{aligned}
\left.2 D_{m} \frac{\partial}{\partial \beta}\left\langle e^{\beta Q_{1}}\right\rangle\right|_{\beta=0} & =1-\sigma_{m}^{z}, \\
\left.2 D_{m}^{2} \frac{\partial^{2}}{\partial \beta^{2}}\left\langle e^{\beta Q_{1}}\right\rangle\right|_{\beta=0} & =\left(1-\sigma_{1}^{z}\right)\left(1-\sigma_{m+1}^{z}\right),
\end{aligned}
$$

где символом $D_{m}$ обозначена производная на решетке $D_{m} f(m)=f(m+1)-$ $f(m)$. При выводе последнего из соотношений (8.13) следует учесть трансляционную инвариантность корреляционных функций.

Мы же рассмотрим еще одну важную корреляционную функцию [27], которая носит название вероятности образования пустоты и может быть получена в пределе $\beta \rightarrow-\infty$. На языке обобщенной модели такой предел производящей функции $\left\langle e^{\beta Q_{1}}\right\rangle$ имеет смысл вероятности обнаружить систему в состоянии, в котором на отрезке $[0, x]$ нет частиц. В случае $X X Z$-цепочки мы получаем вероятность отсутствия квазичастиц в узлах $1, \ldots, m$, иными словами, все спины в этих узлах направлены вверх. Так как мы будем рассматривать корреляционные функции в основном состоянии, которое инвариантно относительно 
одновременного поворота всех спинов, то вероятность обнаружить в первых $m$ узлах все спины вверх равна вероятности обнаружить в первых $m$ узлах все спины вниз. Последняя реализуется в пределе $\beta \rightarrow \infty$. Для того чтобы вычислить этот предел, удобно перейти от переменной $\beta$ к переменной $\varkappa=e^{\beta}$. Легко видеть, что оператор $e^{\beta Q_{1}}$ оказывается полиномом от $\varkappa=e^{\beta}$ степени $m$ :

$$
e^{\beta Q_{1}}=\prod_{k=1}^{m}\left(\frac{1+\varkappa}{2}+\frac{1-\varkappa}{2} \sigma_{k}^{z}\right), \quad \varkappa=e^{\beta} .
$$

Тогда вероятность образования пустоты дается формулой

$$
\mathscr{P}(m)=\lim _{\beta \rightarrow \infty} e^{-m \beta}\left\langle e^{\beta Q_{1}}\right\rangle=\left\langle\prod_{k=1}^{m}\left(\frac{1-\sigma_{k}^{z}}{2}\right)\right\rangle .
$$

\section{9. Вероятность образования пустоты в пределе $M \rightarrow \infty$}

До сих пор под вектором $|\psi(\{\lambda\})\rangle$ мы подразумевали некоторое собственное состояние трансферматрицы. Для вычисления корреляционных функций в термодинамическом пределе $(M \rightarrow \infty)$ при нулевой температуре в качестве вектора $|\psi(\{\lambda\})\rangle$ следует выбрать состояние, отвечающее минимальному собственному значению гамильтониана (8.1) (основное состояние). Здесь сразу следует сделать оговорку по поводу неоднородной модели $X X Z$, которую мы ввели в предыдущем разделе и которую продолжаем рассматривать. Как уже отмечалось, неоднородная модель при произвольных значениях параметров неоднородности $\xi_{k}$ не имеет физического содержания, поэтому говорить об основном состоянии в такой модели вообще бессмысленно. Однако если количество параметров неоднородности $\xi_{k}$, отличных от $\eta / 2$, в пределе $M \rightarrow \infty$ остается конечным, то свойства такой модели в термодинамическом пределе совпадают со свойствами обычной однородной $X X Z$-цепочки (8.1). Для наших целей вполне достаточно будет считать различными параметры $\xi_{k}$, соответствующие первым $m$ узлам, а все остальные положить равными $\eta / 2$.

Следует также оговорить конкретную фазу модели, в которой мы собираемся вычислять вероятность образования пустоты. В зависимости от значения параметра анизотропии $\Delta$ в $X X Z$-цепочке при $M \rightarrow \infty$ существуют три различные фазы [2], [7]. При $\Delta \leqslant-1$ основное состояние системы является ферромагнитным и все корреляционные функции становятся тривиальными. Физический интерес представляет случай $\Delta>-1$, когда основное состояние оказывается антиферромагнитным, однако и здесь следует рассматривать две возможности $\Delta>1$ и $-1<\Delta \leqslant 1$. В первом случае возбужденные состояния отделены от основного конечной щелью (массивная фаза), во втором случае щель отсутствует (безмассовая фаза). Мы рассмотрим термодинамический предел в безмассовой фазе, имея в виду дальнейшее применение полученных формул к конкретному случаю $\Delta=1 / 2$. Поскольку $\Delta=\operatorname{ch} \eta$, удобно сделать замену $i \eta=\zeta, 0 \leqslant \zeta<\pi$, и тогда $\Delta=\cos \zeta$. 
Полагая $\{\mu\}=\{\lambda\}$ в формуле (7.16) и нормируя это выражение с помощью условия (6.22), мы получаем для производящей функции $X X Z$-цепочки

$$
\left\langle e^{\beta Q_{1}}\right\rangle=\frac{(-1)^{N}}{N !} \oint_{\Gamma(\{\lambda\})} \prod_{j=1}^{N}\left(\frac{d z_{j}}{2 \pi i} \cdot \frac{l\left(z_{j}\right)}{l\left(\lambda_{j}\right)}\right) \cdot \frac{\left[\operatorname{det}_{N} \Omega_{\varkappa}\left(z_{k}, \lambda_{j}\right)\right]^{2}}{\prod_{j=1}^{N} \mathscr{Y}_{\varkappa}\left(z_{j} \mid\{z\}\right) \cdot \operatorname{det}_{N} \frac{\partial \mathscr{Y}\left(\lambda_{j} \mid\{\lambda\}\right)}{\partial \lambda_{k}}},
$$

где $\varkappa=e^{\beta}$. Напомним, что контуры интегрирования $\Gamma(\{\lambda\})$ не содержат никаких сингулярностей подынтегрального выражения, кроме точек $\lambda_{j}$, которые для основного состояния при $-1<\Delta \leqslant 1$ являются вещественными числами. Легко видеть, что помимо полюсов при $z_{k}=\lambda_{j}$ подынтегральная функция имеет сингулярности только в решениях твистованных уравнений Бете $\prod_{j=1}^{N} \mathscr{Y}_{\varkappa}\left(z_{j} \mid\{z\}\right)=0$. Для того чтобы гарантированно отделить эти особенности от полюсов при $z_{k}=\lambda_{j}$, мы будем считать $\varkappa$ достаточно малым, а параметры неоднородностей $\xi_{k}$ близкими к $-i \zeta / 2$. В этом случае можно показать (см. [37]), что все решения твистованных уравнений Бете лежат в малых окрестностях $\xi_{k}+i \zeta$, т. е. в окрестности $i \zeta / 2$. В то же время условие малости $\varkappa$ не накладывает каких-либо существенных ограничений, поскольку, как мы видели, производящая функция $e^{\beta Q_{1}}$ является полиномом по $\varkappa$, и, следовательно, для определения этого полинома достаточно найти его в окрестности любой наперед заданной точки.

Формально, написанный интеграл равен сумме вычетов в полюсах $z_{k}=\lambda_{j}$, однако их вычисление вызывает серьезные технические трудности, особенно с учетом того, что часть из упомянутых полюсов двойные. Преобразуем интеграл (9.1) путем нескольких последовательных деформаций контуров интегрирования [40].

Подынтегральное выражение является $i \pi$-периодической функцией по каждой переменной $z_{k}$, поэтому сумма вычетов в полюсах внутри контуров интегрирования равна сумме вычетов в полюсах вне контуров интегрирования, умноженной на $(-1)^{N}$. Как мы уже отмечали, единственными особенностями вне контуров $\Gamma(\{\lambda\})$ являются решения твистованных уравнений Бете $\prod_{j=1}^{N} \mathscr{Y}_{\varkappa}\left(z_{j} \mid\{z\}\right)=0$. В окрестности этих решений мы можем заменить один из детерминантов $\operatorname{det} \Omega_{\varkappa}$ на $\operatorname{det} \Omega$, воспользовавшись равенством (6.28). Тогда получим

$$
\left\langle e^{\beta Q_{1}}\right\rangle=\frac{1}{N !} \oint_{\mathrm{TBE}} \prod_{j=1}^{N}\left(\frac{d z_{j}}{2 \pi i} \cdot \frac{l\left(z_{j}\right)}{l\left(\lambda_{j}\right)}\right) \cdot \frac{\operatorname{det}_{N} \Omega_{\varkappa}\left(z_{k}, \lambda_{j}\right) \cdot \operatorname{det}_{N} \Omega\left(\lambda_{k}, z_{j}\right)}{\prod_{j=1}^{N} \mathscr{Y}_{\varkappa}\left(z_{j} \mid\{z\}\right) \cdot \operatorname{det}_{N} \frac{\partial \mathscr{Y}\left(\lambda_{j} \mid\{\lambda\}\right)}{\partial \lambda_{k}}},
$$

где интегрирование ведется вокруг решений твистованных уравнений Бете.

Вновь перейдем к вычислению интеграла по вычетам вне контуров интегрирования. После замены $\operatorname{det} \Omega_{\varkappa}$ на $\operatorname{det} \Omega$ структура особенностей вне контуров изменилась. Поскольку матрица $\Omega$ не имеет полюсов при $z_{k}=\lambda_{j}$, мы имеем теперь лишь простые полюсы в этих точках, обусловленные особенностями $\operatorname{det} \Omega_{\varkappa}$. Кроме того, в подынтегральном выражении появились новые полюсы функций $l\left(z_{k}\right)$ в точках $z_{k}=\xi_{j}, j=1, \ldots, m$. Таким образом, мы приходим 
к следующему выражению:

$$
\begin{aligned}
\left\langle e^{\beta Q_{1}}\right\rangle=\frac{(-1)^{N}}{N !} \oint_{\Gamma(\{\lambda\}) \cup \Gamma(\{\xi\})} \prod_{j=1}^{N}\left(\frac{d z_{j}}{2 \pi i} \cdot \frac{l\left(z_{j}\right)}{l\left(\lambda_{j}\right)}\right) \\
\quad \times \frac{\operatorname{det}_{N} \Omega_{\varkappa}\left(z_{k}, \lambda_{j}\right) \cdot \operatorname{det}_{N} \Omega\left(\lambda_{k}, z_{j}\right)}{\prod_{j=1}^{N} \mathscr{Y}_{\varkappa}\left(z_{j} \mid\{z\}\right) \cdot \operatorname{det}_{N} \frac{\partial \mathscr{Y}\left(\lambda_{j} \mid\{\lambda\}\right)}{\partial \lambda_{k}}},
\end{aligned}
$$

где контуры интегрирования охватывают точки $z_{k}=\lambda_{j}$ и $z_{k}=\xi_{j}$. На этот раз все полюсы подынтегрального выражения оказываются простыми, и интеграл достаточно легко вычисляется. В пределе $\varkappa \rightarrow \infty$ мы получаем для вероятности образования пустоты

$$
\begin{aligned}
\mathscr{P}(m)= & \sum_{\alpha \cup \bar{\alpha}} \frac{\prod_{a \in \alpha} \prod_{b=1}^{m} \operatorname{sh}\left(\lambda_{a}-\xi_{b}\right) \operatorname{sh}\left(\lambda_{a}-\xi_{b}+\eta\right)}{\prod_{a \in \alpha} \prod_{b \in \alpha} \operatorname{sh}\left(\lambda_{a}-\lambda_{b}+\eta\right)} \\
& \times \frac{\operatorname{det}_{j \in \alpha} t\left(\lambda_{j}, \xi_{k}\right) \cdot \operatorname{det}_{j \in \alpha} \tilde{\rho}\left(\lambda_{j}, \xi_{k}\right)}{\prod_{a, b=1, a \neq b}^{m} \operatorname{sh}\left(\xi_{a}-\xi_{b}\right) \cdot \prod_{j \in \alpha} \hat{\rho}\left(\lambda_{j}\right)} .
\end{aligned}
$$

Здесь функция $\hat{\rho}(\lambda)$ определена в $(6.24)$, а функция $\tilde{\rho}\left(\lambda_{j}, \xi_{k}\right)$ определяется с помощью матрицы, обратной к матрице (6.23):

$$
\tilde{\rho}\left(\lambda_{j}, \xi_{k}\right)=-\sum_{\ell=1}^{N} \mathscr{R}_{j \ell} t\left(\lambda_{\ell}, \xi_{k}\right),
$$

где

$$
\sum_{\ell=1}^{N}\left(\delta_{j \ell}-\frac{K\left(\lambda_{j}-\lambda_{\ell}\right)}{\hat{\rho}\left(\lambda_{\ell}\right)}\right) \mathscr{R}_{\ell k}=\delta_{j k} .
$$

Поясним кратко основные шаги, приводящие к формуле (9.4) (более подробное изложение можно найти в работах [37], [45], [46]). После вычисления интеграла мы получаем сумму по разбиениям набора $\{\lambda\}=\left\{\lambda_{\alpha}\right\} \cup\left\{\lambda_{\bar{\alpha}}\right\}$, а также сумму по разбиениям набора $\{\xi\}$. Благодаря свойству $a^{-1}\left(\xi_{j}\right)=0($ см. (8.7)) зависимость от $\varkappa$ в получившейся формуле оказывается полиномиальной, и мы получаем возможность перейти к пределу $\varkappa \rightarrow \infty$. В этом пределе в сумме по разбиениям набора $\{\xi\}$ выживает лишь одно слагаемое, пропорциональное $\varkappa^{m}$. Наконец, матрица $\tilde{\rho}\left(\lambda_{j}, \xi_{k}\right)$ возникает в результате деления детерминанта $\operatorname{det}_{N} \Omega\left(\lambda_{k}, z_{j}\right)$ на детерминант $\operatorname{det}_{N}\left(\partial \mathscr{Y}\left(\lambda_{j} \mid\{\lambda\}\right) / \partial \lambda_{k}\right)$.

В формуле (9.4) уже несложно перейти к термодинамическому пределу. В пределе $M \rightarrow \infty$ параметры $\{\lambda\}$ заполняют всю вещественную ось. Если упорядочить $\lambda_{j}$ в порядке возрастания, то можно показать, что $\lambda_{j+1}-\lambda_{j} \sim M^{-1}$ при $M \rightarrow \infty$. Система уравнений Бете (4.6) переходит в линейное интегральное уравнение на спектральную плотность распределения $\rho(\lambda)[7]$ :

$$
-2 \pi i \rho(\lambda)+\int_{-\infty}^{\infty} K(\lambda-\mu) \rho(\mu) d \mu=t\left(\lambda,-\frac{i \zeta}{2}\right),
$$



где

$$
\rho\left(\lambda_{j}\right)=\lim _{M \rightarrow \infty} \frac{1}{M\left(\lambda_{j+1}-\lambda_{j}\right)} .
$$

Уравнение (9.7) явно решается с помощью преобразования Фурье, и мы получаем

$$
\rho(\lambda)=\frac{1}{2 \zeta \operatorname{ch}(\pi \lambda / \zeta)} .
$$

Теперь, чтобы получить окончательную формулу для вероятности образования пустоты, нам следует перейти в выражении (9.4) от суммирования по параметрам $\lambda$ к интегрированию, с учетом формул $(9.7),(9.9)$. Легко видеть, что функция $\hat{\rho}(\lambda)$ в пределе $M \rightarrow \infty$ связана со спектральной плотностью распределения $\rho(\lambda)$ следующим простым соотношением:

$$
\lim _{M \rightarrow \infty} \frac{\hat{\rho}(\lambda)}{M}=-2 \pi i \rho(\lambda)
$$

Матрица $\mathscr{R}_{j k}$ в пределе превращается в резольвенту интегрального оператоpa (9.7), что позволяет явно вычислить функцию $\tilde{\rho}\left(\lambda, \xi_{k}\right)$ :

$$
\tilde{\rho}\left(\lambda, \xi_{k}\right) \rightarrow \frac{i}{2 \zeta \operatorname{sh}\left(\pi\left(\lambda-\xi_{k}\right) / \zeta\right)}, \quad M \rightarrow \infty
$$

Наконец, переходя от суммирования по разбиениям к интегрированию, мы получаем для вероятности образования пустоты в термодинамическом пределе следующее выражение [46]:

$$
\begin{aligned}
\mathscr{P}(m) & =\frac{1}{m ! \prod_{a, b=1, a \neq b}^{m} \operatorname{sh}\left(\xi_{a}-\xi_{b}\right)} \int_{-\infty}^{\infty} d^{m} \lambda \prod_{a=1}^{m} \prod_{b=1}^{m} \frac{\operatorname{sh}\left(\lambda_{a}-\xi_{b}\right) \operatorname{sh}\left(\lambda_{a}-\xi_{b}-i \zeta\right)}{\operatorname{sh}\left(\lambda_{a}-\lambda_{b}-i \zeta\right)} \\
& \times \operatorname{det}_{m}\left(\frac{-i \sin \zeta}{\operatorname{sh}\left(\lambda_{j}-\xi_{k}\right) \operatorname{sh}\left(\lambda_{j}-\xi_{k}-i \zeta\right)}\right) \cdot \operatorname{det}_{m}\left(\frac{1}{2 \zeta \operatorname{sh}\left(\pi\left(\lambda-\xi_{k}\right) / \zeta\right)}\right) \cdot
\end{aligned}
$$

\section{0. Вероятность образования пустоты при $\Delta=1 / 2$}

В ряде случаев кратный интеграл (9.12) может быть вычислен точно. Один из таких случаев возникает при $\Delta=0$. При этом $m$-кратный интеграл факторизуется на произведение $m$ обычных интегралов, и конечный ответ для вероятности образования пустоты дается в виде детерминанта матрицы Тёплица порядка $m$. Можно показать, однако, что при $\Delta=0$ модель эквивалентна свободным фермионам, поэтому корреляционные функции такой модели не представляют собой слишком большого интереса. В любом случае при $\Delta=0$ существуют гораздо более простые методы вычисления корреляционных функций, быстро приводящие к искомому результату [15], [20], [21].

Другой точно решаемый случай возникает при значении параметра анизотропии $\Delta=1 / 2$. С точки зрения физики точка $\Delta=1 / 2$ является точкой общего положения в безмассовой фазе. Тем более любопытно, что в этой точке 
интеграл (9.12) также факторизуется, причем конечный ответ получается даже проще, нежели в случае свободных фермионов [47].

Положим в формуле (9.12) $\zeta=\pi / 3$ и, воспользовавшись представлением для детерминанта Коши (6.17), после несложных преобразований получим

$$
\begin{aligned}
& \mathscr{P}(m)=\left(\frac{3 i}{4 \pi}\right)^{m} \frac{(-1)^{\left(m^{2}-m\right) / 2}}{2^{m^{2}} m !} \prod_{a>b}^{m} \frac{\operatorname{sh} 3\left(\xi_{b}-\xi_{a}\right)}{\operatorname{sh}\left(\xi_{b}-\xi_{a}\right)} \prod_{\substack{a, b=1 \\
a \neq b}}^{m} \frac{1}{\operatorname{sh}\left(\xi_{a}-\xi_{b}\right)} \\
& \quad \times \int_{-\infty}^{\infty} d^{m} \lambda \operatorname{det}_{m}\left(\frac{1}{\operatorname{sh}\left(\lambda_{j}-\xi_{k}+i \pi / 3\right)}\right) \operatorname{det}_{m}\left(\frac{1}{\operatorname{sh}\left(\lambda_{j}-\xi_{k}\right) \operatorname{sh}\left(\lambda_{j}-\xi_{k}-i \pi / 3\right)}\right) .
\end{aligned}
$$

Видно, что $m$-кратный интеграл уже факторизован. Действительно, в силу симметрии подынтегрального выражения по переменным $\lambda_{j}$, мы можем заменить один из детерминантов в (10.1) на произведение диагональных членов, после чего внести каждый сомножитель в соответствующую строку второго детерминанта:

$$
\begin{aligned}
\mathscr{P}(m)= & \left(\frac{3 i}{4 \pi}\right)^{m} \frac{(-1)^{\left(m^{2}-m\right) / 2}}{2^{m^{2}}} \prod_{a>b}^{m} \frac{\operatorname{sh} 3\left(\xi_{b}-\xi_{a}\right)}{\operatorname{sh}\left(\xi_{b}-\xi_{a}\right)} \prod_{\substack{a, b=1 \\
a \neq b}}^{m} \frac{1}{\operatorname{sh}\left(\xi_{a}-\xi_{b}\right)} \\
& \times \operatorname{det}_{m}\left(\int_{-\infty}^{\infty} \frac{d \lambda_{j}}{\operatorname{sh}\left(\lambda_{j}-\xi_{k}\right) \operatorname{sh}\left(\lambda_{j}-\xi_{k}-i \pi / 3\right) \operatorname{sh}\left(\lambda_{j}-\xi_{k}+i \pi / 3\right)}\right) .
\end{aligned}
$$

Поскольку в конечном итоге при переходе к однородному пределу нам предстоит устремить все $\xi_{k} \mathrm{\kappa}-i \pi / 6$, мы положим $\xi_{k}=\varepsilon_{k}-i \pi / 6$ и будем считать, что переменные $\varepsilon_{k}$ находятся в достаточно малой окрестности нуля. Тогда интеграл в (10.2) однозначно определен, и после частичного перехода к пределу $\varepsilon_{k} \rightarrow 0$ мы находим

$$
\begin{aligned}
\mathscr{P}(m)= & \frac{(-1)^{\left(m^{2}-m\right) / 2} 3^{\left(m^{2}+m\right) / 2}}{2^{m^{2}}} \\
& \times \lim _{\varepsilon_{\ell} \rightarrow 0} \frac{1}{\prod_{a, b=1, a \neq b}^{m} \operatorname{sh}\left(\varepsilon_{a}-\varepsilon_{b}\right)} \cdot \operatorname{det}\left(\frac{\operatorname{sh}\left(\left(\varepsilon_{j}-\varepsilon_{k}\right) / 2\right)}{\operatorname{sh}\left(3\left(\varepsilon_{j}-\varepsilon_{k}\right) / 2\right)}\right) .
\end{aligned}
$$

Оставшийся предел в формуле (10.3) не зависит от того, как именно переменные $\varepsilon_{k}$ достигают своего предельного значения. Более того, легко показать, что

$$
\begin{aligned}
& \lim _{\varepsilon_{\ell} \rightarrow 0} \prod_{\substack{a, b=1 \\
a \neq b}}^{m} \frac{1}{\left(\varepsilon_{a}-\varepsilon_{b}\right)} \cdot \operatorname{det}\left(\frac{\operatorname{sh}\left(\left(\varepsilon_{j}-\varepsilon_{k}\right) / 2\right)}{\operatorname{sh}\left(3\left(\varepsilon_{j}-\varepsilon_{k}\right) / 2\right)}\right) \\
& \quad=\lim _{\substack{\varepsilon_{\ell} \rightarrow 0 \\
\varepsilon_{\ell}^{\prime} \rightarrow 0}} \prod_{a>b}^{m} \frac{1}{\operatorname{sh}\left(\varepsilon_{a}-\varepsilon_{b}\right) \operatorname{sh}\left(\varepsilon_{b}^{\prime}-\varepsilon_{a}^{\prime}\right)} \cdot \operatorname{det}_{m}\left(\frac{\operatorname{sh}\left(\left(\varepsilon_{j}-\varepsilon_{k}^{\prime}\right) / 2\right)}{\operatorname{sh}\left(3\left(\varepsilon_{j}-\varepsilon_{k}^{\prime}\right) / 2\right)}\right),
\end{aligned}
$$


где мы ввели дополнительный набор переменных $\varepsilon_{k}^{\prime}$. Тогда, полагая $\varepsilon_{j}=\epsilon j$ и $\varepsilon_{k}^{\prime}=\epsilon(1-k)$, мы получим

$$
\begin{aligned}
\mathscr{P}(m)= & (-1)^{\left(m^{2}-m\right) / 2} 3^{\left(m^{2}+m\right) / 2} \\
& \times \lim _{\epsilon \rightarrow 0} \prod_{j>k}^{m}\left(\frac{1}{\operatorname{sh} \epsilon(j-k)}\right)^{2} \cdot \operatorname{det}_{m}\left(\frac{\operatorname{sh}(\epsilon(j+k-1) / 2)}{\operatorname{sh}(3 \epsilon(j+k-1) / 2)}\right) .
\end{aligned}
$$

Детерминант в формуле (10.5) уже вычисляется явно. Действительно, при произвольных комплексных $\alpha$ и $\beta$ имеем [48]

$$
\frac{1}{\prod_{j>k}^{m} \operatorname{sh}^{2} \beta(j-k)} \cdot \operatorname{det} \frac{\operatorname{sh} \alpha(j+k-1)}{\operatorname{sh} \beta(j+k-1)}=2^{m^{2}-m} \prod_{j=1}^{m} \prod_{k=1}^{m} \frac{\operatorname{sh}(\alpha+\beta(j-k))}{\operatorname{sh} \beta(j+k-1)} .
$$

Остается подставить в эту формулу $\alpha=\epsilon / 2, \beta=3 \epsilon / 2$, после чего переход к пределу $\epsilon \rightarrow 0$ становится тривиальным. Окончательно получаем

$$
\mathscr{P}(m)=\left(\frac{1}{2}\right)^{m^{2}} \prod_{k=0}^{m-1} \frac{(3 k+1) !}{(m+k) !} .
$$

Таким образом, вероятность образования пустоты при значении параметра анизотропии $\Delta=1 / 2$ полностью вычислена. Любопытным является тот факт, что числа

$$
A_{m}=\prod_{k=0}^{m-1} \frac{(3 k+1) !}{(m+k) !}
$$

хорошо известны в комбинаторике и представляют собой числа матриц чередующихся знаков порядка $m$. Тесная связь между $X X Z$-цепочкой при $\Delta=1 / 2$ и матрицами чередующихся знаков подмечена сравнительно недавно [49] и до настоящего времени не получила еще исчерпывающего объяснения.

\section{Заключение}

В настоящем обзоре мы постарались продемонстрировать, каким образом результаты, полученные в рамках абстрактной алгебраической модели, можно использовать для вычисления корреляционных функций конкретных квантовомеханических систем. Мы рассмотрели лишь один пример алгебры (2.2) с $R$-матрицей вида (2.8). Однако уже одна эта алгебра описывает такие интересные физические модели, как $X X Z$-цепочка Гейзенберга, модели одномерного Бозе-газа и синус-Гордон. Во всех этих моделях полученные алгебраические результаты позволяют (при надлежащем определении действия локальных операторов на векторы пространства состояний) получать интегральные представления для корреляционных функций. Последние уже могут быть использованы для вычисления физических характеристик квантовых систем, например получения асимптотического поведения корреляторов при больших временах и расстояниях. 
Алгебраический анзац Бете, как метод построения собственных функций гамильтонианов, применим и в случае более сложных $R$-матриц, например для эллиптической $R$-матрицы восьмивершинной модели Бакстера [50], [51]. Однако вопрос об использовании этого метода для вычисления корреляционных функций соответствующих физических моделей изучен еще недостаточно хорошо.

\section{Список литературы}

[1] H. Bethe, "Zur Theorie der Metalle. I. Eigenwerte und Eigenfunktionen der linearen Atomkette", Z. Phys., 71:3-4 (1931), 205-226.

[2] L. Hulthén, "Über das Austauschproblem eines Kristalls", Ark. Mat. Astron. Fysik, 26, № 11, 1938, 1-106.

[3] R. Orbach, "Linear antiferromagnetic chain with anisotropic coupling", Phys. Rev., 112:2 (1958), 309-316.

[4] L. R. Walker, "Antiferromagnetic linear chain", Phys. Rev., 116:5 (1959), 1089-1090.

[5] E. H. Lieb, W. Liniger, "Exact analysis of an interacting Bose gas. I. The general solution and the ground state", Phys. Rev., 130:4 (1963), 1605-1616; E. H. Lieb, "Exact analysis of an interacting Bose gas. II. The exitation spectrum", Phys. Rev., 130:4 (1963), 1616-1624.

[6] E. H. Lieb, D. C. Mattis (eds.), Mathematical physics in one dimension: exactly soluble models of interacting particles, Academic Press, New York, 1966.

[7] C. N. Yang, C. P. Yang, "One-dimensional chain of anisotropic spin-spin interactions. I. Proof of Bethe's hypothesis for ground state in a finite system", Phys. Rev., 150:1 (1966), 321-327; "II. Properties of the ground state energy per lattice site in a finite system", 327-339.

[8] C. N. Yang, "Some exact results for the many-body problem in one dimension with repulsiv delta-function interaction", Phys. Rev. Lett., 19:23 (1967), 1312-1315.

[9] J. des Cloizeaux, M. Gaudin, "Anisotropic linear magnetic chain", J. Math. Phys., 7:8 (1966), 1384-1400.

[10] M. Gaudin, La fonction d'onde de Bethe, Collect. Commissariat Energ. Atom. Ser. Sci., Masson, Paris, 1983; рус. пер.: М. Годен, Волновая функиия Бете, Мир, М., 1987.

[11] Ф.А. Березин, В.Н. Сушко, "Релятивистская двумерная модель самовзаимодействующего поля ненулевой массы покоя”, ЖЖЭТФ, 45 (1965), 1293-1306; англ. пер.: F.A. Berezin, V.N. Sushko, "Relativistic two-dimensional model of a self-interacting fermion field with non-vanishing rest mass", Soviet Phys. JETP, 21 (1965), 865.

[12] E. H. Lieb, F. Y. Wu, "Absence of Mott transition in an exact solution of the short range one band model in one dimension", Phys. Rev. Lett., 20:25 (1968), 1445-1448.

[13] L. Onsager, "Cristal statistics. I. A two-dimensional model with an order-disorder transition", Phys. Rev., 65:3-4 (1944), 117-149.

[14] T. T. Wu, B. M. McCoy, C. A. Tracy, E. Barouch, "Spin-spin correlation functions for the two-dimensional Ising model: exact theory in the scaling region", Phys. Rev. B, 13:1 (1976), 316-374.

[15] B. M. McCoy, C. A. Tracy, T. T. Wu, "Two-dimensional Ising model as an exactly solvable relativistic quantum field theory: explicit formulas for $n$-point functions", Phys. Rev. Lett., 38:15 (1977), 793-796.

[16] E. Lieb, T. Shultz, D. Mattis, "Two soluble models of an antiferromagnetic chain", Ann. Physics, 16:3 (1961), 407-466. 
[17] B. M. McCoy, "Spin correlation functions of the $X-Y$ model", Phys. Rev., 173:2 (1968), 531-541.

[18] A. Lenard, "Momentum distribution in the ground state of the one-dimensional system of impenetrable bosons", J. Math. Phys., 5:7 (1964), 930-943.

[19] A. Lenard, "One-dimensional impenetrable bosons in thermal equilibrium", J. Math. Phys., 7:7 (1966), 1268-1272.

[20] H. G. Vaidya, C.A. Tracy, "One particle reduced density matrix of impenetrable bosons in one dimension at zero temperature", Phys. Rev. Lett., 42:1 (1979), 3-6.

[21] Ф. Коломо, А. Г. Изергин, В.Е.Корепин, В. Тогнетти, “Температурные корреляционные функции в XXО-цепочке Гейзенберга. I", ТМФ, 94:1 (1993), 19-51; англ. пер.: F. Colomo, A. G. Izergin, V. E. Korepin, V. Tognetti, "Temperature correlation functions in the XX0 Heisenberg chain. I", Theoret. and Math. Phys., 94:1 (1993), $11-38$.

[22] Л.А. Тахтаджян, Л.Д. Фаддеев, "Квантовый метод обратной задачи и $X Y Z$ модель Гейзенберга", УМH, 34:5 (1979), 13-63; англ. пер.: L. A. Takhtadzhan, L. D. Faddeev, "The quantum method of the inverse problem and the Heisenberg XYZ model", Russian Math. Surveys, 34:5 (1979), 11-68.

[23] Е. К. Склянин, Л. А. Тахтаджян, Л. Д. Фаддеев, "Квантовый метод обратной задачи. I", ТМФ, 40:2 (1979), 194-220; англ. пер.: E. K. Sklyanin, L. A. Takhtadzhyan, L. D. Faddeev, "Quantum inverse problem method. I", Theoret. and Math. Phys., 40:2 (1979), 688-706.

[24] L. D. Faddeev, "How algebraic Bethe ansatz works for integrable models", Symétries quantiques (Les Houches, France, 1995), eds. A. Connes et al., North-Holland, Amsterdam, 1998, 149-219.

[25] N. Kitanine, J. M. Maillet, V. Terras, "Form factors of the $X X Z$ Heisenberg spin-1/2 finite chain", Nuclear Phys. B, 554:3 (1999), 647-678; arXiv: math-ph/9807020.

[26] P. P. Kulish, E. K. Sklyanin, "Quantum spectral transform method. Recent developments", Integrable quantum field theories (Tvärminne, Finland, 1981), Lecture Notes in Phys., 151, eds. J. Heitarinta, C. Montonen, Springer, Berlin-New York, 1982, 61-119.

[27] A. G. Izergin, V.E. Korepin, "The quantum inverse scattering method approach to correlation functions", Comm. Math. Phys., 94:1 (1984), 67-92.

[28] V.E. Korepin, "Calculation of norms of Bethe wave functions", Comm. Math. Phys., 86:3 (1982), 391-418.

[29] В.Е. Корепин, “Анализ билинейного соотношения шестивершинной модели”, ДАН СССР, 265:6 (1982), 1361-1364; англ. пер.: V. E. Korepin, "Analysis of the bilinear relation of a six-vertex model”, Soviet Phys. Dokl., 27:12 (1982), 1050-1051.

[30] А. Г. Изергин, "Статистическая сумма шестивершинной модели в конечном объеме", Докл. АН СССР, 297:2 (1987), 331-333; англ. пер.: A. G. Izergin, "Partition function of a six-vertex model in a finite volume", Soviet Phys. Dokl., 32:11 (1987), 878-879.

[31] L. D. Faddeev, "History and perspectives of quantum groups", Milan J. Math., 74:1 (2006), 279-294.

[32] V.E. Korepin, N. M. Bogoliubov, A. G. Izergin, Quantum inverse scattering method and correlation functions, Cambridge Monogr. Math. Phys., Cambridge Univ. Press, Cambridge, 1993.

[33] Е. К. Склянин, "Метод обратной задачи рассеяния и квантовое нелинейное уравнения Шрёдингера", Докл. АН СССР, 244:6 (1979), 1337-1340; англ. пер.: E. K. Sklyanin, "Method of the inverse scattering problem and the nonlinear quantum Schrödinger equation", Soviet Phys. Dokl., 24 (1979), 107-109.

[34] Е. К. Склянин, Л. Д. Фаддеев, "Квантовомеханический подход к вполне интегрируемым моделям теории поля”, Докл. АН СCCP, 243:6 (1978), 1430-1433; англ. 
пер.: E. K. Sklyanin, L.D. Faddeev, "Quantum mechanical approach to completely integrable field theory models", Soviet Phys. Dokl., 23 (1978), 902-904.

[35] A. G. Izergin, V.E. Korepin, "Pauli principal for one-dimensional bosons and the algebraic Bethe anstaz", Lett. Math. Phys., 6:4 (1982), 283-288.

[36] V. Tarasov, A. Varchenko, "Bases of Bethe vectors and difference equations with regular singular points", Internat. Math. Res. Notices, 1995, № 13, 637-669.

[37] N. Kitanine, J. M. Maillet, N. A. Slavnov, V. Terras, "Master equation for spin-spin correlation functions of the $X X Z$ chain", Nucl. Phys. B, 712:3 (2005), 600-622; arXiv: hep-th/0406190.

[38] Н.А. Славнов, "Вычисление скалярных произведений волновых функций и формфакторов в алгебраическом анзаце Бете", ТMФ, 79:2 (1989), 232-240; англ. пер.: N. A. Slavnov, "Calculation of scalar products of wave functions and form factors in the framework of the alcebraic Bethe ansatz", Theoret. and Math. Phys., 79:2 (1989), 502-508.

[39] Н.А. Славнов, "Об одном тождестве для дуальных полей", Записки науч. сем. ПОМИ, 245 (1997), 270-281; англ. пер.: N. A. Slavnov, "On an identity for dual fields", J. Math. Sci. (New York), 100:2 (2000), 2181-2188.

[40] N. Kitanine, K. Kozlowski, J. M. Maillet, N. A. Slavnov, V. Terras, "On correlation functions of integrable models associated with the six-vertex $R$-matrix", J. Stat. Mech., 2007, № 1, P01022; arXiv: hep-th/0611142.

[41] W. Heisenberg, "Zur Theorie des Ferromagnetismus", Z. Phys., 49:9-10 (1928), 619-636.

[42] P. P. Kulish, E. K. Sklyanin, "Quantum inverse scattering method and the Heisenberg ferromagnet", Phys. Lett., 70:5-6 (1979), 461-463.

[43] П. П. Кулиш, "Квантовый метод обратной задачи и точно решаемые модели статистической физики", Труды II Международного симпозиума по избранным проблемам статистической физики, Дубна, 1981, 147-157.

[44] A. G. Izergin, V.E. Korepin, "Correlation functions for the Heisenberg XXZ-antiferromagnet", Comm. Math. Phys., 99:2 (1985), 271-302.

[45] N. Kitanine, J. M. Maillet, N. A. Slavnov, V. Terras, "Spin-spin correlation functions of the $X X Z-1 / 2$ Heisenberg chain in a magnetic field", Nucl. Phys. B, 641:3 (2002), 487-518; arXiv: hep-th/0201045.

[46] N. Kitanine, J. M. Maillet, V. Terras, "Correlation functions of the $X X Z$ Heisenberg spin-1/2 chain in a magnetic field", Nucl. Phys. B, 567:3 (2000), 554-582; arXiv: math-ph/9907019.

[47] N. Kitanine, J. M. Maillet, N. A. Slavnov, V. Terras, "Emptiness formation probability of the $X X Z$ spin-1/2 Heisenberg chain at $\Delta=1 / 2$ ", J. Phys. A, 35:27 (2002), L385-L388; arXiv: hep-th/0201134.

[48] G. Kuperberg, "Another proof of the alternating sign matrix conjecture", Internat. Math. Res. Notices, 1996, № 3, 139-150; arXiv: math.C0/9712207.

[49] A. Razumov, Yu. Stroganov, "Spin chains and combinatorics", J. Phys. A, 34:14 (2001), 3185-3190; arXiv: cond-mat/0012141.

[50] R. J. Baxter, "Partition function of the eight-vertex lattice model", Ann. Physics, 70:1 (1972), 193-228.

[51] R. J. Baxter, "Eight-vertex model in lattice statistics and one-dimensional anisotropic Heisenberg chain. I. Some fundamental eigenvectors", Ann. Physics, 76:1 (1973), 1-24; "II. Equivalence to a generalized ice-type lattice model", 25-47; "III. Eigenvectors of the transfer matrix and hamiltonian", 48-71. 